\title{
Contractual Externalities and Common Agency Equilibria*
}

\author{
David Martimort** and Lars Stole***
}

First version: May 1999

Revised version: February 2003

\begin{abstract}
This paper characterizes the equilibrium sets of common agency games with direct externalities between principals when they compete with nonlinear prices. Direct externalities arise when the contracting variable of one principal directly affects the other principal's payoff. First, we characterize the set of pure-strategy, symmetric equilibria under complete information of an intrinsic common agency game. This set of equilibria is large because of the presence of priceoutput offers by the principals that are unchosen by the agent in equilibrium. Equilibria exist in which principals offer out-of-equilibrium price-output choices to the agent and induce aggressive, low-price behavior corresponding to marginal-cost pricing in the extreme case. We then show that this equilibrium set of outputs is robust to the possibility that agent refuses any of the offered contracts; the case of delegated agency. Second, we introduce asymmetric information in order to rationalize existing nonlinear pricing contracts. The introduction of asymmetric information has the effect of restricting the set of equilibrium outputs of the intrinsic common agency game.
\end{abstract}

JEL Classification Nos.: D82, L51.

Keywords: Common Agency, Externality, Adverse Selection, Equilibrium Selection.

* We thank Sandeep Baliga, Drew Fudenberg, Jean-Jacques Laffont and Thibaud Vergé for helpful comments. The Editor of this journal, Patrick Bolton, and two referees have been instrumental in helping us to improve the paper. All errors are ours.

** Université de Toulouse 1 (IDEI-GREMAQ) and Institut Universitaire de France, FRANCE.

*** University of Chicago, GSB, Chicago, U.S.A. 


\section{Introduction}

In many economic contexts several principals contract with a common agent and each of those principals is directly affected by the actions selected by the common agent to the other principals. In such a context, there are direct externalities among the principals. In this paper, we are interested in describing the equilibrium set of such simple common agency (or "'bidding") games; i.e., the set of outputs which result from a non-cooperative behavior between the principals when the latter competes through nonlinear prices. We distinguish and analyze two variations of the common agency game - the intrinsic common agency game and the delegated common agency game, to use the language of Bernheim and Whinston (1986a). These games differ with respect to the outside option of the common agent. Under intrinsic common agency, the agent can either accept the whole set of contracts offered by the principals or reject all of the contracts. Under delegated common agency, the agent can also choose a strict subset of principals for whom he wants to work.

A typical example of such common agency games with externalities is that of two retailers who distribute the output of a common manufacturer and compete on a final market. Both retailers (the principals) independently and non-cooperatively contract with the common manufacturer (the agent) and the production put on the final market by one retailer affects the price received by the other. Another example would be that of competing bidders submitting bid schedules to a common seller in an externality context where the quantity bought by one bidder affects the profit of another. Multi-unit auctions for electricity capacities or UMTS auctions are two real-world examples.

We are particularly interested in the shape of the nonlinear prices that principals offer in the equilibria of those games and on the consequences of that shape both in terms of allocations (which outputs are produced) but also in terms of distribution (how much the agent gets).

Following the tradition of standard principal-agent models, we characterize the set of implementable allocations, i.e., the set of allocations obtained as pure-strategy Nash equilibria of the common agency game with deterministic mechanisms. We first focus on the simplest case of intrinsic common agency where the agent can only accept both contracts or none. Under 
complete information, we find a large set of such equilibria. Remarkably, any output between the Cournot and competitive outcomes can be sustained as part of an equilibrium of the intrinsic common agency game in which principals compete through nonlinear prices. This multiplicity is driven by the tariff choices available to the agent that are unchosen in equilibrium. This points at a first role of nonlinear prices in common agency games: including such out-of-equilibrium offers in their respective tariffs has a commitment value. The principals are able to change their behaviors on the final good market and become more aggressive. This out-of-equilibrium effect of contracts has no equivalent in a monopolistic screening environment and can only be achieved through offering nonlinear price schedules with options that are unchosen by the agent. Such extensions of tariff schedules to include more than the equilibrium choices of the agent can generate intense competition between the principals, serving as implicit threats to prevent rival principals from deviating from the equilibrium allocation. In the case of complete information, a pure-strategy equilibrium requires that the agent chooses a single transfer-output allocation from each principal, hence any offer richer than a single transfer-output offer (e.g., a nonlinear price schedule) must contain unchosen offers. Without such out-of-equilibrium offers, the Cournot outcome is the only equilibrium result of the intrinsic common agency game under complete information. Thus, out-of-equilibrium offers can only intensify competition between the principals. ${ }^{1}$

Under delegated agency, the agent has the additional choices of selecting only one principal's contract and rejecting the other's. In that case, we demonstrate an even greater importance of out-of-equilibrium choices. When principals are forced to use singleton contract offers (i.e., direct revelation mechanisms) rather than menus of offers in a delegated common agency, the only purestrategy equilibrium (when it exists) is for head-to-head competition for the right of exclusive agency; principals earn zero profits. With a more realistic extension of the strategy spaces to allow for nonlinear prices, more collusive outcomes can be sustained where principals share the market. Any output between Cournot and the competitive outcome is still an equilibrium of

\footnotetext{
${ }^{1}$ As a by-product of this analysis, common agency environments with direct externalities offer a clear failure of the naive Revelation Principle which would suggest that it is without loss of generality to look for truthful equilibria in a game in which each principal offers direct mechanisms. Under complete information, the agent's type space is degenerate and the restriction to direct mechanisms is equivalent to a restriction to singleton offers rather than menus of offers. This restriction is with loss of generality. A complete discussion of these types of failures can be found in Martimort and Stole (2002).
} 
the delegated common agency game with such extensions. This result implies that equilibrium output sets are equivalent under intrinsic and delegated common agency games with complete information. This finding also suggests a second role of out-of-equilibrium offers: such offers change the strategic nature of the game, making it possible to move from intense (head-to-head) competition to more collusive outcomes.

The multiplicity of equilibrium outcomes that exist under complete information arise because out-of-equilibrium offers are costless for a principal to include. Under complete information (and along the equilibrium path), only one transfer-output pair from each principal is chosen by the agent. The remaining portions of a principal's nonlinear price schedule are irrelevant for the offering principal's payoffs; they only impact the rival principal's strategy. Hence, such unchosen out-of-equilibrium extensions are hard to justify in a complete information world, especially under intrinsic agency where the equilibrium with no out-of-equilibrium offers Pareto dominates all other equilibria from the principals' collective viewpoint. With this motivation, we introduce private information. It is well known that nonlinear prices are useful for monopoly principals when their agent has private information; the same is true for competing duopoly principals. By introducing private information, we introduce a cost to arbitrary nonlinear pricing, and indicate a more standard, role of non-linear prices. We are particularly interested in looking at how this introduction interacts with the commitment role of contracts stressed earlier.

To introduce asymmetric information in its simplest form, we assume that some parameter of the agent's cost function is unknown to both principals. We then derive the symmetric and differentiable pure-strategy equilibria of the intrinsic common agency game. We find that adverse selection introduces incentive compatibility constraints that restrict the slope of the equilibrium tariff at all equilibrium outputs. The freedom under complete information in specifying an extension of the equilibrium nonlinear price schedule is greatly reduced by these constraints and the set of equilibrium outputs correspondingly shrinks. The screening role of contract somewhat contradicts their commitment value.

Section 2 presents our common agency model. Section 3 analyzes the case of complete information and intrinsic common agency. Section 4 deals with the case of delegated common 
agency game under complete information. Section 5 characterizes the equilibrium set with intrinsic common agency and asymmetric information. Section 7 reviews the literature. Section 8 concludes. All proofs are relegated to an Appendix.

\section{A common agency model with direct externalities}

Two retailers (the principals, $i=1,2$ ) sell perfect substitutes on a final market. Each unit of final good $q_{i}$ is produced from one unit of an intermediate good produced by a single manufacturer (the common agent wholesaler). The principals are assumed to have all bargaining power in offering contracts to the retailer, the implicit assumption here is that there are a large number of potential manufacturers available to produce the inputs used by retailers. ${ }^{2}$

The common agent has a quasi-linear utility function:

$$
U=y-\theta C(Q),
$$

where $y$ is a monetary transfer, $Q=q_{1}+q_{2}$ is the total production of the manufacturer, and $\theta$ is a parameter reflecting the cost efficiency of this common agent. $C(\cdot)$ is the agent's cost function which is strictly increasing and convex and has a positive third derivative $\left(C^{\prime}(\cdot)>0\right.$ and $C^{\prime \prime}(\cdot)>0$ with $\left.C^{\prime \prime \prime}(\cdot)>0\right)$ and such that $C(0)=0$. For technical reasons, we assume that the Iñada condition $C^{\prime}(0)=0$ holds. Inverse demand on the principals' market for the homogeneous final good is denoted by $P(Q)$ with $0<P(0)<\bar{\theta}, P^{\prime}(\cdot)<0,\left|P^{\prime}(0)\right|<+\infty$ and $P^{\prime \prime}(\cdot) \leq 0$. Without loss of generality, we assume that the retailer $i$ produces final output at no additional cost. Principal $i$ 's profit is thus given by:

$$
V_{i}\left(t_{i}, q_{i}, q_{-i}\right)=P\left(q_{i}+q_{-i}\right) q_{i}-t_{i}
$$

when he buys a quantity $q_{i}$ at price $t_{i}$ and his rival $j \neq i$ puts a quantity $q_{j}$ on the final market. Note that the agent's production contracted upon by the rival principal $j$ enters directly into principal $i$ 's objective function. We thus have an instance of direct contractual externalities. This introduction of direct externalities into common agency settings with adverse selection stands in sharp contrast with the models developed by Stole (1991) and Martimort (1992). In

\footnotetext{
${ }^{2}$ Segal and Whinston (2001) analyze a related model where bargaining power may be more evenly distributed.
} 
these latter papers, we both assumed that contractual externalities between the principals occur only because the agent's marginal utility for contractual activity $i$ depends also on contractual activity $j \neq i$, and not that this activity, $q_{j}$, enters directly into $i$ 's utility function.

We assume that principal $i$ can only contract with the common agent on activity $q_{i}$ and not on the production for the rival principal, $q_{j}$. One possible justification for this assumption is that the principal may neither have the auditing rights nor the monitoring technologies to observe and, thus, contract on $q_{j}$. A second justification is that the retail price may not be contractible for the retailers either because resale price maintenance is not allowed or because the retail price reflects non-verifiable after-sales services. ${ }^{3}$ In the same way, principal $i$ cannot contract on principal $j$ 's contract, because the latter contract is not observable by principal $i .{ }^{4}$

A pure-strategy, deterministic contract between principal $i$ and the agent is thus a nonlinear schedule, $T_{i}\left(q_{i}\right)$, mapping the agent's choice of output into the transfer paid by principal $i .^{5}$ Applying a generalization of the Taxation Principle, Martimort and Stole (2002) show that there is no loss of generality in considering this class of deterministic nonlinear prices if the modeler is interested in common agency equilibria with deterministic mechanisms. The essence of this Taxation Principle is that extending nonlinear prices to allow further messages on top of quantity choices is of no additional value. From the agent's point of view, communication-per-se is of no value, only the payoff-relevant consequences of such communication matter. Hence, communication can be replaced with a decentralized menu of choices that are undominated. For example, in the seemingly more general game in which offered nonlinear price schedules depend upon the messages sent by the agent, we could replace the mechanism with the upper envelope of transfers without any strategic consequences for either the principals or the agent. Since pure-strategy equilibria have a natural economic appeal, we further restrict our attention to pure-strategy equilibria in deterministic nonlinear prices. ${ }^{6}$

\footnotetext{
${ }^{3}$ See Tirole (1988, p. 177) for a justification of this assumption.

${ }^{4}$ Katz (1987) shows in the related context of delegation games between principal-agent pairs that such reciprocal conditioning of contracts may imply the nonexistence of a contract equilibrium.

${ }^{5}$ These mechanisms are deterministic since the agent chooses an output and not a distribution of outputs among which the principal could randomize. Not only do these randomizations seem unrealistic in the real world and hardly observed in the case of nonlinear pricing, but they also require that judicial courts can enforce these randomizations. This may be quite problematic since any deviation away from the randomization which has been contracted upon can only be detected statistically after many such realizations of the contract. Such detections are instead not feasible in the one-shot relationships that we analyze here.

${ }^{6}$ Nonetheless, even within this class of equilibria, we find that out-of-equilibrium messages play a significant
} 
We normalize the common agent's reservation utility to zero if he decides not to produce. In a first step of our analysis, we consider a model of intrinsic common agency in which the agent produces simultaneously for both retailers or for none. We will also test the robustness and investigate whether the same equilibrium outputs emerge under delegated common agency, i.e., when the agent may also choose to work with only one of the competing retailers. In this case, the agent has the extra option of working for a single principal and that may affect the transfers realized with either principal.

The intrinsic common agency game unfolds as follows:

1. First, nature draws $\theta$. This parameter is known only by the common agent under the case of asymmetric information or by all players under complete information.

2. Principals (non-cooperatively) offer nonlinear price schedules.

3. The common agent accepts or refuses both contracts.

4. If he refuses, the common agent gets his reservation utility normalized at zero. If he accepts, he chooses how much to produce for each principal.

In the case of a delegated common agency game, stage 3 of the timing above is replaced by

3'. The common agent chooses to accept both, one, or none of the contracts.

A delegated common agency equilibrium arises if the agent gets more utility by taking both contracts than by taking only one of the contracts. In other words, an allocation is a delegated common agency equilibrium only if it is immune to deviations either by a principal or by the agent where a principal is exclusively served by the common agent.

In what follows, it is useful to define the following output allocations as relevant benchmarks under complete information on $\theta$ :

role in describing the set of equilibrium outcomes. It is noteworthy that because mixed-strategy equilibria are also limits of pure-strategy equilibria of games of incomplete information between the principals, the pure-strategy equilibria of such Bayesian common agency games could be obtained using the same techniques as in the present paper. 
- For a given $\theta$, the competitive symmetric output, $q^{b}(\theta)$, satisfies:

$$
P\left(2 q^{b}(\theta)\right)=\theta C^{\prime}\left(2 q^{b}(\theta)\right)
$$

The price of one unit of final good equals then its marginal cost of production.

- The Cournot symmetric output, $q^{c}(\theta)$, satisfies:

$$
P\left(2 q^{c}(\theta)\right)+q^{c}(\theta) P^{\prime}\left(2 q^{c}(\theta)\right)=\theta C^{\prime}\left(2 q^{c}(\theta)\right)
$$

The marginal revenue of each competing retailer equals then the agent's marginal cost of production.

- The monopoly symmetric output per firm, $q^{m}(\theta)$, satisfies:

$$
P\left(2 q^{m}(\theta)\right)+2 q^{m}(\theta) P^{\prime}\left(2 q^{m}(\theta)\right)=\theta C^{\prime}\left(2 q^{m}(\theta)\right) .
$$

Note that this is the fictional per-firm "monopoly" output; a single monopolist firm would procure $2 q^{m}(\theta)$ in aggregate. In that case, the marginal revenue made by a coalition of both retailers equals the agent's marginal cost of production. This is the outcome achieved had the principals cooperated in their contractual offers to the common agent.

It is immediate to check that $q^{m}(\theta)<q^{c}(\theta)<q^{b}(\theta)$ as a result of a greater exercise of market power on the final good market when one varies outcomes from competitive, to Cournot, and then to monopoly. Moreover, the Iñada assumption $C^{\prime}(0)=0$ and the facts that $P(0)>0$ and $\left|P^{\prime}(0)\right|<\infty$ ensures that $q^{b}(\theta), q^{c}(\theta)$ and $q^{m}(\theta)$ remain all positive. Finally, we assume that aggregate profits are positive for those outputs. This means that:

$$
q^{i}(\theta) P\left(2 q^{i}(\theta)\right) \geq \frac{\theta}{2} C\left(2 q^{i}(\theta)\right)
$$

for $i=b, c, m$.

\section{Intrinsic common agency under complete information}

We start by analyzing the equilibria of the intrinsic common agency game in a setting of complete information in which both principals know the value of $\theta$. Hence, we are interested in equilibria in which, for a fixed $\theta$, the principals play a game in designing optimal nonlinear price schedules 
for the agent. To be clear, we make this dependence on $\theta$ explicit, characterizing the transfers as functions of output, conditional on the commonly known $\theta: T_{i}\left(q_{i} \mid \theta\right)$. When we consider the asymmetric information game in which transfers are designed without knowledge of $\theta$, this dependence is absent: $T_{i}\left(q_{i}\right)$.

\subsection{Multiplicity of equilibria}

We completely characterize the set of symmetric equilibria of the intrinsic common agency game which are sustained with twice-differentiable nonlinear prices defined over a sufficiently large domain of outputs. From here on, we will use the phrase symmetric, differentiable equilibrium, to imply these restrictions.

To determine retailer 1's optimal choice of transfer, $T_{1}\left(q_{1} \mid \theta\right)$, let us take as given the nonlinear price $T_{2}\left(q_{2} \mid \theta\right)$ offered by principal 2 to the common agent. This allows us to define the agent's optimal choice of $q_{2}$, given that $q_{1}$ is supplied to retailer 1 .

$$
q_{2}^{*}\left(q_{1}, \theta\right) \equiv \arg \max _{q_{2}} T_{2}\left(q_{2} \mid \theta\right)-\theta C\left(q_{1}+q_{2}\right)
$$

Assuming that the maximand above is sufficiently regular such that its optimal solution is uniquely characterized by a first-order condition, we can define $q_{2}^{*}\left(q_{1}, \theta\right)$ as: ${ }^{7}$

$$
T_{2}^{\prime}\left(q_{2}^{*}\left(q_{1}, \theta\right) \mid \theta\right)=\theta C^{\prime}\left(q_{1}+q_{2}^{*}\left(q_{1}, \theta\right)\right) \quad \forall \quad\left(\theta, q_{1}\right)
$$

Under complete information, principal 1's best response to the schedule $T_{2}(\cdot \mid \theta)$ offered by principal 2 consists in implementing a production $q_{1}(\theta)$ and a transfer $t_{1}(\theta)=T_{1}\left(q_{1}(\theta) \mid \theta\right)$ which are solutions to the following problem:

$$
\max _{\left\{t_{1}, q_{1}\right\}} P\left(q_{1}+q_{2}^{*}\left(q_{1}, \theta\right)\right) q_{1}-t_{1}
$$

\footnotetext{
${ }^{7}$ In the Appendix, we check that equilibrium nonlinear prices can be designed so that the agent's program is indeed concave. Provided that these transfer functions are defined over a broad domain to exclude corner solutions, the first-order approach above is valid. To characterize the common agent's behavior with a first-order condition, we first assume that the nonlinear tariff $T_{j}\left(q_{j} \mid \theta\right)$ offered by principal $j \neq i$ is differentiable and second the agent's maximization program is concave. Henceforth, we focus a priori on common agency equilibria of the intrinsic common agency game with nonlinear tariffs which satisfy these conditions, and then we check ex post that the derived equilibrium tariffs do indeed justify our working assumption. In fact, the first-order approach is valid for all outputs $q_{i}$, i.e., both for outputs on and off the equilibrium path. Under delegated common agency, we will have to be more cautious, the tariffs constructed to sustain the equilibrium outputs will not be such that the agent's behavior is always characterized with a first-order condition.
} 
subject to

$$
t_{1}+T_{2}\left(q_{2}^{*}\left(q_{1} \mid \theta\right), \theta\right)-\theta C\left(q_{1}+q_{2}^{*}\left(q_{1}, \theta\right)\right) \geq 0,
$$

where $q_{2}^{*}\left(q_{1}, \theta\right)$ is defined through the first-order condition (1). (2) is the agent's participation constraint which stipulates that the agent has to accept both contracts (rather than none) in the intrinsic common agency game. Note, of course, that the problem above determines the equilibrium values of the output and transfer but leave undetermined the options which remain unchosen by the agent. Those options will be precisely constructed to prevent deviations by the other principal.

The following proposition characterizes the full-information pure-strategy equilibrium of our intrinsic common agency game with deterministic and twice-differentiable nonlinear tariffs.

Proposition 1 : Under complete information, every (and only) output $q(\theta) \in\left[q^{c}(\theta), q^{b}(\theta)\right]$ is part of a symmetric, differentiable equilibrium of the intrinsic common agency game.

Moreover, in all such equilibria, the agent gets zero rent.

$$
U(\theta)=2 T(q(\theta) \mid \theta)-\theta C(2 q(\theta))=0 .
$$

Proposition 1 establishes that the intrinsic common agency game under complete information is plagued with a large set of equilibria. The reason for this multiplicity is simple. Let us fix $\theta$ which is known to all players of the game. By offering a smooth nonlinear price schedule for this value of $\theta$, principal $i$ not only controls the agent's equilibrium production of intermediate good $q_{i}$ but also how the agent behaves around this equilibrium point following any unexpected contractual offer made by principal $j \neq i$. This extra control of the agent's behavior off-theequilibrium path changes the degree of the principals' competition on the final market. Choices offered by the principals that are not taken in equilibrium by the agent constrain the principals from inducing the agent to produce a different output than that conjectured in equilibrium.

The intuition for the fact that competition between the principals raises output above the monopoly outcome is rather straightforward. Indeed, since the common agent can always substitute away production for principal $i$ against production for principal $j$, each principal pays 
at the margin too much for the good he buys from the agent. In equilibrium, the agent thus increases the sales for each principal with respect to a situation where the principals would have cooperated. Too much output is placed on the final market from the principals' perspectives. The contractual externality between the principals is positive in marginal returns to output, resulting in excessive activities in both relationships.

In any equilibrium, a principal does not choose output below the equilibrium value because of the threat that the agent will sell enough production to his rival to make any such attempt of raising the price-cost margin on his units unprofitable. A lesser degree of competition is obtained when the agent has little incentive to substitute one production against the other. This occurs when both principals offer a relatively steep nonlinear prices around the equilibrium value of output. In the extreme case, the quantity produced for the non-deviating principal remains fixed following any unexpected offer and the Cournot outcome is implemented. At the other extreme, the maximal degree of competition is obtained when productions for each principal can be substituted one-for-one. In such a case, no principal can decrease output below the equilibrium value without triggering a one-for-one substitution by the agent. As a result, the retail price is fixed at its competitive value and the competitive outcome is implemented. By varying the slope of the out-of-equilibrium transfer-output pairs, any output between the extremes of Cournot and competitive can be implemented.

More generally, different equilibrium outputs correspond to different slopes of the symmetric nonlinear equilibrium tariff around these equilibrium points. Indeed, by offering a nonlinear price schedule which is conveniently extended outside the set of equilibrium outputs, principal $j$ can control principal $i$ 's incentives to capture the final market. This is done at the contracting stage by stipulating with extra transfer-output choices how the agent's production for principal $j$ should respond when principal $i$ deviates from his equilibrium offer. As argued in the proof of Proposition 1 and stated in the corollary below, different degrees of convexity of these equilibrium nonlinear prices correspond to different equilibrium beliefs on the degree of competition that arises on the final market. Equilibrium outputs which are close to the competitive outcome correspond to the least cooperative outcomes and to the flattest tariffs around the equilibrium point. Equilibrium outputs which are close to the Cournot outcome correspond instead to the 
least competitive outcomes and the steepest schedules around the equilibrium point. In this case, principal $i$ has passive beliefs about how the agent changes his production of intermediate good $q_{j}$ following any unexpected offer he receives from principal $i$. The Cournot outcome is the most cooperative outcome achieved through bilateral contracting. We show in the Appendix that imposing the optimality of the agent's decision problem, together with the first-order conditions of the principals' programs, forces at least this minimal amount of competition between the principals.

In the proof of Proposition 1 we demonstrate that quadratic nonlinear price schedules are sufficient to implement the output set. From this it follows that we can parameterize the equilibrium outputs by the value of the second derivative of the symmetric nonlinear price $T^{\prime \prime}(q \mid \theta)=\gamma$ at the equilibrium point $q=q(\theta)$.

Corollary 1 : Under complete information, any symmetric equilibrium output $q(\theta)$ is such that:

$$
q(\theta) P^{\prime}(2 q(\theta))+P(2 q(\theta))=\theta C^{\prime}(2 q(\theta))+\frac{q(\theta) P^{\prime}(2 q(\theta))}{1-\frac{\gamma}{\theta C^{\prime \prime}(2 q(\theta))}},
$$

for some $\gamma \in(-\infty, 0]$.

The symmetric nonlinear price $T(q \mid \theta)$ which implements the equilibrium output $q(\theta)$ above can be taken to be quadratic and then writes as

$$
T(q \mid \theta)=\frac{\theta}{2} C(2 q(\theta))+\theta C^{\prime}(2 q(\theta))(q-q(\theta))+\frac{\gamma}{2}(q-q(\theta))^{2} .
$$

Note that $\gamma=0$ for the competitive outcome. Any incentives of a deviating principal to reduce his own output is then offset by an extra production for the non-deviating principal as it can be seen on the first-order condition (1) which then becomes $2 q^{b}(\theta)=q_{1}+q_{2}^{*}\left(q_{1}, \theta\right)$. On the other hand, $\gamma=-\infty$ corresponds to an infinitely steep nonlinear price at the equilibrium point and this yields the Cournot outcome.

Note that changing $\gamma$ modifies the commitment value of the contract. Of course, in the present framework, choosing any $\gamma$ is costless for the principal. In Section 5, we will introduce 
asymmetric information to restore a cost of changing the curvature of the nonlinear price because it has now a screening role also.

\subsection{Restrictions to singleton contract offers}

It may seem odd a priori to use a whole nonlinear price schedule to control the agent in a world of complete information. One might consider, instead, restricting attention to contracts which are singletons: $\left\{t_{i}, q_{i}\right\}$. Given that such degenerate contracts have the same dimensionality as the agent's (for now) degenerate type space, we sometimes refer to these singleton offers as direct mechanisms. By definition, singleton contracts (i.e., direct mechanisms) cannot contain offers that go unused in equilibrium. Nonlinear tariffs, in contrast, allow the agent of known type $\theta$ the opportunity to choose among a continuum of contract offers and there is not a one one-to-one mapping between each item on a principal's contract menu and the agent's type. The extra flexibility incorporated in the out-of-equilibrium extensions of the equilibrium nonlinear price seems an artificial device to construct many equilibrium outcomes. Even if those extensions are consistent with equilibrium behavior, one may cast some doubts on equilibria if they are sustained with what may look like "non-credible" threats.

To see this point more clearly, let us come back on the description of the set of equilibria of Proposition 1. The more competitive outcomes are sustained with the threat that each principal, say principal 1 , believes that his rival will induce a very aggressive behavior from the agent if principal 1 tries to deviate from the equilibrium output. Of course, this threat is not credible if we explicitly introduce the possibility for principal 2 to further react to principal 1's deviation. Without being explicit on the extensive form of such a game, it should be clear that the only equilibrium outcome of such a process of reactions and counter actions is the Cournot outcome. ${ }^{8}$ This Cournot outcome, which is the lower bound of the equilibrium set, can also be implemented as an equilibrium outcome of a common agency game played with singleton contract offers (i.e., direct revelation mechanisms) in this complete information setting.

In view of Proposition 1, singleton contract offers are not particularly attractive since they are far from being enough to describe the whole equilibrium set. However, one can also justify

\footnotetext{
${ }^{8}$ The equilibrium concept required here is similar to the reactive equilibrium of Riley (1979).
} 
the use of such contracts if there exists some menu cost or more generally some cost of writing contingencies. ${ }^{9}$ If such costs are large enough, principals will only make offers that are chosen in equilibrium. In this case, competition between the principals leads to a unique outcome.

Proposition 2 : The Cournot output $q^{c}(\theta)$ is the unique equilibrium of the intrinsic common agency game under complete information when principals are restricted to singleton contracts of the form $\left\{t_{i}, q_{i}\right\}$.

This result is not very surprising in view of previous discussions. With a singleton contract, principal 2 cannot control the agent's behavior off the equilibrium path for any unexpected contractual offer made by principal 1 . The common agent does not change his production of intermediate $q_{2}$ following such an unexpected offer. Expectations about the agent's behavior off the equilibrium path are passive beliefs. Obviously, these passive beliefs implement the same outcome as that obtained with extended nonlinear prices which are very steep around the equilibrium point.

The comparison of Propositions 1 and 2 also confirms the unavailability of the Revelation Principle (at least a version of it with deterministic mechanisms). The set of equilibrium outcomes achieved with richer mechanisms that allow principals' menus to constrain offers which are unchosen in equilibrium differs significantly from the set of equilibrium outcomes achieved when both principals are restricted to use singleton contracts (direct mechanisms). This latter result reinforces a finding of Martimort and Stole (1998) where we analyze less structured games between competing principals. ${ }^{10}$ Focusing on these direct revelation mechanisms involves a quite important loss of generality even in meaningful economic environments. ${ }^{11}$

The multiplicity of equilibria obtained with unchosen equilibrium menu items and complete information only arises because we have considered a setting with direct externalities between the principals who compete on the final market. Let us instead suppose that retailers have exclusive

\footnotetext{
${ }^{9}$ Of course, this argument to justify simple direct mechanisms in our setting is a bit contrived. After all, the description of the whole equilibrium set is obtained with simple quadratic schedules which can be defined with only three parameters.

${ }^{10}$ See also Peck (1996) for a related example in the case of a multiprincipal-multiagent model.

${ }^{11}$ The fundamental reason for the failure of the Revelation Principle is that direct mechanisms are, by definition, unable to convey information on how a principal would like the agent to react to a deviation made by the other principal. In the vocabulary of Epstein and Peters (1999), direct mechanisms based on the agent's report of only his exogenous preference information are unable to convey market information.
} 
territories and thus are local monopolies on segmented markets. The only interaction between the retailers comes now from their use of a common retailer. We are in the framework of the earlier common agency literature due to Stole (1991) and Martimort $(1992,1996)$ which involves no direct externalities. In this case, the principals have no reason to precommit themselves to affect their competition since there is no scope such a competition. The equilibrium outcome under complete information is the same whether principals offer nonlinear prices or only direct mechanisms.

To confirm this result, let us consider a slightly modified version of our basic model in which principals are monopolies on segmented markets. The inverse demand on each market is now $P\left(q_{i}\right)$. Let us now also redefine the monopoly symmetric output, $\tilde{q}^{m}(\theta)$, as:

$$
P\left(\tilde{q}^{m}(\theta)\right)+\tilde{q}^{m}(\theta) P^{\prime}\left(\tilde{q}^{m}(\theta)\right)=\theta C^{\prime}\left(2 \tilde{q}^{m}(\theta)\right) .
$$

This output maximizes the aggregate payoff of the coalition made of both principals and the agent, namely $q_{1} P\left(q_{1}\right)+q_{2} P\left(q_{2}\right)-\theta C\left(q_{1}+q_{2}\right)$.

Proposition 3 Under complete information and without direct externalities, the unique purestrategy symmetric equilibrium of the intrinsic common agency game achieves the monopoly outcome, $q(\theta)=\tilde{q}^{m}(\theta)$. It can be implemented with singleton contracts.

Without direct externalities, the equilibrium contract offered by retailer 1 to the common agent takes into account that the latter optimally adapts his production of intermediate good $q_{1}$ to any change in $q_{2}$ induced by retailer 2's deviation. A small change in $q_{2}$ away from the equilibrium value has now only a second-order effect on the aggregate payoff of the coalition between retailer 1 and the agent since output $q_{2}$ does not affect directly retailer 1's payoff. Hence, retailer 1 has no incentive to use the agent's behavior as an implicit veto threat against any possible deviation by retailer 2 . In this case, singleton contracts are sufficient to describe the whole equilibrium set, which is itself a singleton.

On the contrary, in the case of direct externalities, a small change in $q_{2}$ away from the equilibrium value has also a first-order effect on the payoff of the coalition made of retailer 1 and the common agent through the change in $q_{1}^{*}\left(q_{2}, \theta\right)$ it induces. Offered, but unchosen menu 
items available to the agent generate equilibria where the principals behave more (respectively, less) aggressively on the final good market than with direct mechanisms when this externality is positive (respectively, negative). It is important to communicate market information on how the principals behave for unexpected outputs and this new kind of communication can only be achieved with offers that are not selected by the agent in equilibrium.

\section{Delegated common agency under complete information}

We now move to the case where the agent can choose to take only one of the equilibrium contracts offered by the principal in addition to the choices of both contracts and non-participation. This possibility generally leaves greater surplus to the agent and increases the strategic complexity of the game. The greater complexity has two sources. First, to sustain a delegated common agency equilibrium, it must be that principal $i$ offers to the agent more when he takes both contracts than what he gets by taking only principal $j \neq i$ 's contract. Second, it must be that principal $i$ does not want to deviate in such a way that the agent no longer serves principal $j$. In this sense, the existence of common (rather than exclusive) agency is determined in equilibrium and is not exogenously fixed.

It is useful to begin with the simpler strategy space of singleton contracts in the delegation game. To this end, we first consider singleton contracts (direct mechanisms) in the delegated agency games, i.e., we restrict each of the principals to offering a single transfer-output pair, $\left\{t_{i}(\theta), q_{i}(\theta)\right\}$. Such mechanisms do not allow for any offers to remain unchosen in equilibrium; in other words, there are no out-of-equilibrium messages, to use the language of mechanism design. After analyzing this simple setting, we turn to nonlinear transfer-output schedules which allow for out-of-equilibrium (i.e., unchosen) offers.

\subsection{Singleton contracts}

To illustrate the role of out-of-equilibrium messages in this context, it is useful to first analyze what happens when unchosen offers are not allowed.

Proposition 4 Equilibria in complete information, delegated common agency games with sin- 
gleton contracts:

- There does not exist any pure-strategy equilibrium in which the agent serves both principals;

- If

$$
2 q^{m}(\theta) P\left(2 q^{m}(\theta)\right)-\theta\left(C\left(4 q^{m}(\theta)\right)-C\left(2 q^{m}(\theta)\right)\right)<0,
$$

then there exists a pure-strategy equilibrium of the delegated common agency game in which the agent serves each principal with equal probability, produces $2 q^{m}(\theta)$ and receives an equilibrium transfer $t(\theta)=2 q^{m}(\theta) P\left(2 q^{m}(\theta)\right)$ from the selected principal. The agent gets a strictly positive equilibrium rent equal to

$$
U(\theta)=2 q^{m}(\theta) P\left(2 q^{m}(\theta)\right)-\theta C\left(2 q^{m}(\theta)\right)>0,
$$

and the principals make zero profits.

The intuition behind the non-existence of a common agency equilibrium where both principals are served in equilibrium is the following. Starting from an allocation where both retailers are simultaneously served by the agent, one of the principals would always find it beneficial to deviate upwards and request more output from the common agent so that the latter chooses not to serve the other principal. The mere possibility of not being served by any given principal, say principal 1, can be viewed as a particular extension of the direct mechanism involving a null contractual option $(t, q)=(0,0)$. This option can be used by principal 2 to open new strategic possibilities, inducing the agent to refuse principal 1's contract.

In a pure-strategy equilibrium with direct mechanisms augmented with the $(t, q)=(0,0)$ option, the principals who are identical in all respects compete fiercely for the exclusive service of the agent because each principal wants to induce the agent to take this null option in his rival's contract. Conditional on the agent being exclusive, the preferred output chosen by the selected principal is of course the monopoly output which maximizes the payoff of the coalition between the selected principal and the agent. The game has now a Bertrand flavor but what is auctioned off between the principals is the right to be exclusively served by the agent. Hence, the equilibrium output is not the competitive outcome but instead the monopoly outcome. 
Given that an exclusivity clause cannot be imposed at the outset by the principals, the existence of such an equilibrium with exclusivity can only be guaranteed if the agent does not find it optimal to take both exclusive contracts from the principals simultaneously. This imposes the condition (5), which simply means that the agent does not find it optimal to take both contracts because the benefit of taking a second contract, namely the added transfer received from a second principal $2 q^{m}(\theta) P\left(2 q^{m}(\theta)\right)$ is lower than the incremental cost $\theta\left(C\left(4 q^{m}(\theta)\right)-C\left(2 q^{m}(\theta)\right)\right)$ incurred when serving a second principal. ${ }^{12}$

\subsection{Nonlinear prices and out-of-equilibrium (unchosen) offers}

The non-existence of a common agency equilibrium can nevertheless be restored if the desire of any given principal to increase output and being exclusively served by the agent can be controlled with a convenient extension of the equilibrium schedule offered by the other principal. Compared with Section 3, out-of-equilibrium messages are not only useful to expand the set of feasible equilibrium outputs as before but now also change the strategic nature of the game, transforming it from a head-to-head competition for the right to be exclusively served into a split-award auction where both retailers are simultaneously active in equilibrium.

We now show that there always exist pure-strategy equilibria such that the final market is shared between both principals. Such equilibria implement outputs which are the same as under intrinsic agency. From an allocative point of view, the option of refusing one contract does not affect the equilibrium set. Of course, this new outside option for the agent has some redistributive consequences since it changes the allocation of surplus between the principals and the agent.

Proposition 5 Under complete information, every (and only) output $q(\theta) \in\left[q^{c}(\theta), q^{b}(\theta)\right]$ can be obtained as a symmetric delegated common agency equilibrium sustained with a piece-wise differentiable nonlinear price $T_{d}(q \mid \theta)$ such that $T_{d}(0 \mid \theta)=0$.

\footnotetext{
${ }^{12}$ When (5) does not hold, no pure strategy equilibrium exists but we have not been able to characterize the mixed-strategy equilibria of the game if any.
} 
In all such equilibria, the agent gets a strictly positive rent:

$$
U(\theta)=2 T_{d}(q(\theta) \mid \theta)-\theta C(2 q(\theta))>0 .
$$

The construction of the out-of-equilibrium messages incorporated in the equilibrium nonlinear price is such that any incentive for upward output deviations by either principal disappears. To do so, it is necessary that any incremental production that a deviating principal, say principal 1 , may request from the agent is compensated by a decrease in the revenue the deviating principal may make for those extra-units. This occurs when the agent substitutes enough production for principal 2 to keep the total production high enough and the price on the final market low enough. Of course, keeping the total production large enough cannot be possible if the only extra option available from principal 2 is for the agent to refuse the latter's contract as it was the case in the game restricted to direct mechanisms. Options which are unchosen in equilibrium are now clearly needed to sustain the delegated common agency equilibria.

Once the equilibrium nonlinear price offered by a given principal is conveniently extended downwards towards the null option $(0,0)$ to prevent upwards deviation by the other principal, the only remaining difficulty is to be sure that, in equilibrium, the agent is indifferent between taking both contracts and taking only one of the contracts. This condition is of course needed since, otherwise, a principal can always reduce the transfer he offers to the agent and gain with such a deviation. This last issue is solved by extending the equilibrium nonlinear price upwards in such a way that the agent is just indifferent between taking both contracts and taking only one and producing for the selected principal a quantity much greater than in equilibrium.

Propositions 4 and 5 also show that, when exclusivity cannot be explicitly contracted upon, out-of-equilibrium choices offered to the agent allow the principals to avoid the head-to-head competition and increase their profits. Clearly, for the principals, those extensions allow more collusive outcomes in which the market is shared and positive profits are realized by both competing retailers. In this respect, direct mechanisms are not particularly attractive since they force principals to behave more aggressively than what they can do with conveniently extended mechanisms. 


\section{Intrinsic common agency under asymmetric information}

In Section 3.2, we argued that nonlinear prices may suffer from a credibility problem under complete information. One way to make credible any output-transfer pair offered in equilibrium by either principal is to make sure that this option is relevant for a particular type of the agent. The standard motivation for looking at nonlinear prices comes then from the fact that the principal is unable to discriminate among the different possible types of the agent in a private information self-selection setting.

Under asymmetric information, the extension of a nonlinear price in the neighborhood of an equilibrium output is not, a priori, without cost as it is under complete information. Outputs in this neighborhood are themselves equilibrium outputs for nearby values of the agent's type which arise with some probability. The competing mechanism design problem is now posed in a much more plausible and structured environment. It therefore seems natural to study common agency games under adverse selection in the hope of getting sharper predictions on the equilibrium set. We thus turn to the case of asymmetric information in the framework of our intrinsic common agency game. The agent's efficiency parameter $\theta$ is now his private information. It is drawn from the set $\Theta=[\underline{\theta}, \bar{\theta}]$ according to a common knowledge distribution $F(\cdot)$ with positive density $f(\cdot)$. This distribution satisfies the monotone hazard rate property, $\frac{d}{d \theta}\left(\frac{F(\theta)}{f(\theta)}\right)>0$ for all $\theta \in \Theta$. Because $\theta$ is unobservable to the principals, $T(q)$ cannot be designed as a function of $\theta$; hence, we use $T(q)$ rather than $T(q \mid \theta)$.

\subsection{Computing best-responses}

Before proceeding to a systematic investigation of the pure-strategy equilibria of the common agency game under asymmetric information, we propose a general algorithm which helps to characterize the best-response of a principal to any pure-strategy nonlinear contract offered by his rival.

For any nonlinear price $T_{2}(\cdot)$ offered by principal 2 , there is indeed no loss of generality in looking for principal 1's best-response within the class of singleton contracts (i.e., direct mechanisms) of the form $\left\{t_{1}(\hat{\theta}), q_{1}(\hat{\theta})\right\}$ where $\hat{\theta}$ is the agent's report to principal 1 . Any payoff 
that principal 1 can achieve when he offers a mechanism with some general communication space can also be achieved with such a direct revelation mechanism. Here, we simply apply the standard Revelation Principle to determine the outputs implemented by principal 1 at his best response to a given deterministic nonlinear price $T_{2}(\cdot)$ offered by principal 2 . Of course, in equilibrium, nonlinear prices must be best responses to each other. To validate the first-order approach, we extend the nonlinear schedules offered by both principals for out-of-equilibrium outputs. $^{13}$

However, different nonlinear prices offered by principal 2 affect differently the agent's incentives to produce for principal 1 and therefore principal 1's incentives to distort his consumption of intermediate good 1 for informational reasons. In other words, for a given coalition between a principal and the agent, the trade-off between extraction of the agent's information rent and maximization of the aggregated payoff of this coalition depends on the other contract signed by the agent.

To capture this effect mathematically in a clear manner, let us thus define the agent's indirect utility function vis-à-vis principal 1 as:

$$
u^{1}\left(q_{1}, \theta\right)=\max _{q_{2}} T_{2}\left(q_{2}\right)-\theta C\left(q_{1}+q_{2}\right)
$$

This indirect utility function gives the maximal payoff of an agent with type $\theta$ when his production for principal 1 is $q_{1}$ and when he chooses his output $q_{2}$ optimally. When $T_{2}(\cdot)$ is defined over the whole real line, differentiable and sufficiently concave, $q_{2}^{*}\left(q_{1}, \theta\right)$ is again defined by the first-order condition (1). Note that different nonlinear tariffs $T_{2}(\cdot)$ correspond to different indirect functions $u^{1}\left(q_{1}, \theta\right)$ and therefore to possibly different best responses by principal 1 . For notational ease, we leave the dependence of $u^{1}\left(q_{1}, \theta\right)$ on principal 2's contract implicit.

For a given indirect utility function, finding principal 1's best response to $T_{2}(\cdot)$ is a straightforward task using the methodology of single principal-agent optimal contracting problems. The

\footnotetext{
${ }^{13}$ Note that under asymmetric information the chosen portion of the nonlinear price schedule associated with the equilibrium direct mechanisms $\left\{t_{i}(\theta), q_{i}(\theta)\right\}$ is of the form $t_{i}\left(q_{i}^{-1}\left(q_{i}\right)\right)$ and is defined only over the domain $Q_{i}=$ $\left\{q_{i} \mid q_{i}=q_{i}(\theta)\right.$ for some $\left.\theta\right\}$. Using these limited, non-extended nonlinear prices when computing best responses would require the modeler to compute the principal's benefit of making a subset of types with non-zero measure bunch on the corner of the tariff offered by his rival. Such analysis would require modifying (1) as a characterization of the best choice of the agent for any output chosen in the rival's nonlinear price. Stole (1991) shows that these extensions are not be needed in the case of indirect externalities when there is sufficient concavity present.
} 
standard implementability conditions must be satisfied by this contractual best response. The only difference with standard contracting problems comes from the fact that principal 1 suffers from the direct externality exerted by principal 2's contracting. However, from a technical point of view, the difficulty is to ensure that principal 1's problem is concave since, again this concavity is endogenous and depends on the price schedule $T_{2}(\cdot)$ offered by principal 2 . We will first assume throughout that this concavity is satisfied and we will propose a sufficient condition so that it is so.

For convenience, let us also define the $U(\theta)$ as the common agent's information rent:

$$
U(\theta)=\max _{\hat{\theta} \in \Theta} t_{1}(\hat{\theta})+u^{1}\left(q_{1}(\hat{\theta}), \theta\right) .
$$

It turns out that the implementability conditions of principal 1's best response can be expressed more easily in terms of the information rent-output pair $\left\{U(\theta), q_{1}(\theta)\right\}$ rather than in terms of the transfer-output pair $\left\{t_{1}(\theta), q_{1}(\theta)\right\}$. The following lemma is standard in the self-selection literature.

Lemma 1 : If $u_{q \theta}^{1}\left(q_{1}, \theta\right) \leq 0$ for all $\left(q_{1}, \theta\right)$, a pair $\left\{U(\cdot), q_{1}(\cdot)\right\}$ is implementable if and only if, for all $\theta \in \Theta$, the following two conditions are satisfied:

- first-order condition,

$$
\dot{U}(\theta)=u_{\theta}^{1}\left(q_{1}(\theta), \theta\right)
$$

- second-order condition,

$$
q_{1}(\theta) \text { is non-increasing. }
$$

In addition to choosing the pair $\left\{U(\theta), q_{1}(\theta)\right\}$, the principal also chooses an optimal region of participation for agency, $\left[\underline{\theta}, \theta_{0}\right]$, which may may exclude a positive measure of agents, $\left(\theta_{0}, \bar{\theta}\right]$. These three instruments, $\left\{U(\theta), q_{1}(\theta), \theta_{0}\right\}$, are chosen at a best response by principal 1 is therefore solution to the following program:

$\max _{\left\{U(\theta), q_{1}(\theta), \theta_{0}\right\}} \int_{\underline{\theta}}^{\theta_{0}}\left(P\left(q_{1}(\theta)+q_{2}^{*}\left(q_{1}(\theta), \theta\right)\right) q_{1}(\theta)+T_{2}\left(q_{2}^{*}\left(q_{1}(\theta), \theta\right)\right)-\theta C\left(q_{1}(\theta)+q_{2}^{*}\left(q_{1}(\theta)\right)\right)-U(\theta)\right) f(\theta) d \theta$ subject to (8)-(9) and 


$$
U(\theta) \geq 0 \quad \text { for all } \theta \in\left[\underline{\theta}, \theta_{0}\right]
$$

which is the agent's participation constraint. We will use this program throughout when computing the best response of each principal. Note that we implicitly assume at this stage that any principal wants to serve all the types. We give the condition for which it is so in the next proposition.

The standard single-crossing or Spence-Mirrlees property, $u_{1 \theta}^{1}\left(q_{1}, \theta\right) \leq 0$, which is usually assumed to obtain a well-behaved monotonic solution to principal principal 1's problem, can no longer be postulated a priori. Instead, the implicit dependence of $u^{1}\left(q_{1}, \theta\right)$ on principal 2's contract implies that this single-crossing property is endogenous and may or may not arise at the equilibrium of the common agency game. Nevertheless, we focus on pure strategy equilibria where this single-crossing property emerges in both indirect utility functions vis-à-vis either principal.

\subsection{Equilibria set}

For further references, we define the incomplete-information analogues to $q^{c}(\theta)$ and $q^{m}(\theta)$. There is no corresponding analogue for $q^{b}(\theta)$ because no information rents are captured by firms when competitive outcomes are implemented.

- The virtual Cournot symmetric output as $\tilde{q}^{c}(\theta)$ such that:

$$
P\left(2 \tilde{q}^{c}(\theta)\right)+\tilde{q}^{c}(\theta) P^{\prime}\left(2 \tilde{q}^{c}(\theta)\right)=\left(\theta+\frac{F(\theta)}{f(\theta)}\right) C^{\prime}\left(2 \tilde{q}^{c}(\theta)\right) .
$$

- The virtual monopoly symmetric output as $\tilde{q}^{m}(\theta)$ such that:

$$
P\left(2 \tilde{q}^{m}(\theta)\right)+2 \tilde{q}^{m}(\theta) P^{\prime}\left(2 \tilde{q}^{m}(\theta)\right)=\left(\theta+2 \frac{F(\theta)}{f(\theta)}\right) C^{\prime}\left(2 \tilde{q}^{m}(\theta)\right) .
$$

The first output schedule corresponds to the standard Cournot outcome when costs have been replaced by virtual costs to capture the effect of informational asymmetries between the principals and their agent. The second output schedule corresponds to the monopoly outcome when those virtual costs are counted twice. Under our assumptions on cost and demand, both $\tilde{q}^{c}(\theta)$ and $\tilde{q}^{m}(\theta)$ converge to zero as $\theta$ goes to $\bar{\theta}$. Because the most efficient type is $\underline{\theta}$, there is no incentive distortion for the most efficient type: $\tilde{q}^{c}(\underline{\theta})=q^{c}(\underline{\theta})$ and $\tilde{q}^{m}(\underline{\theta})=q^{m}(\underline{\theta})$. 
Note also that the assumptions $P(0)<+\infty,\left|P^{\prime}(0)\right|<+\infty$ and $C(0)=0$ imply necessarily that $q^{b}(\theta), \tilde{q}^{c}(\theta)$, and $\tilde{q}^{m}(\theta)$ all remain positive for any value of $\theta$. This property will also be satisfied by the equilibrium outputs of the intrinsic common agency game.

We can now state the following proposition which describes the set of common agency symmetric equilibria of the game under asymmetric information.

Proposition 6 : Assume that the concavity of the principals' problem and the agent's singlecrossing property both hold. Consider any solution to the differential equation

$$
\dot{q}(\theta)=-\frac{C^{\prime}(2 q(\theta))\left(P(2 q(\theta))+q(\theta) P^{\prime}(2 q(\theta))-\left(\theta+\frac{F(\theta)}{f(\theta)}\right) C^{\prime}(2 q(\theta))\right)}{\theta C^{\prime \prime}(2 q(\theta))\left(P(2 q(\theta))+2 q(\theta) P^{\prime}(2 q(\theta))-\left(\theta+2 \frac{F(\theta)}{f(\theta)}\right) C^{\prime}(2 q(\theta))\right)},
$$

with the boundary condition $q(\underline{\theta}) \in\left[\tilde{q}^{c}(\underline{\theta}), q^{b}(\underline{\theta})\right]$. Then, $q(\theta)$ is a strictly decreasing and positive output schedule implemented in a symmetric equilibrium of the intrinsic common agency game for all participating agents, $\theta \in\left[\underline{\theta}, \theta_{0}\right] . \theta_{0}$, in turn, is the unique root of

$$
V\left(q\left(\theta_{0}\right), \theta_{0}\right)=q\left(\theta_{0}\right) P\left(2 q\left(\theta_{0}\right)\right)+u^{1}\left(q\left(\theta_{0}\right), \theta_{0}\right)+u_{\theta}^{1}\left(q\left(\theta_{0}\right), \theta_{0}\right) \frac{F\left(\theta_{0}\right)}{f\left(\theta_{0}\right)}=0
$$

if a root exists, and is $\theta_{0}=\bar{\theta}$ otherwise.

The corresponding equilibrium nonlinear price schedule, $T(\cdot)$, is uniquely determined by the boundary condition $T\left(q\left(\theta_{0}\right)\right)=\theta_{0} C\left(2 q\left(\theta_{0}\right)\right) / 2$ and the marginal condition $T^{\prime}(q(\theta))=\theta C^{\prime}(2 q(\theta))$.

When $\frac{\partial^{2} q_{2}^{*}\left(q_{1}, \theta\right)}{\partial q_{1}^{2}} \geq 0$ for the corresponding schedule $T(\cdot)$, the principals' programs are concave, the agent's single-crossing property holds, and the necessary conditions above are also sufficient for characterizing equilibrium outputs.

In any such equilibrium, the agent with type $\theta$ gets an information rent:

$$
U(\theta)=\int_{\theta}^{\theta_{0}} C(2 q(z)) d z \geq 0 .
$$

When

$$
q(\bar{\theta}) P(2 q(\bar{\theta}))-\left(\frac{\bar{\theta}}{2}+\frac{1}{f(\bar{\theta})}\right) C(2 q(\bar{\theta}))>0
$$

both principals want to deal with all types of the common agent: $\theta_{0}=\bar{\theta}$. 
Under asymmetric information, the slope of the symmetric equilibrium nonlinear price $T(q)$ is defined at any equilibrium point $q(\theta)$ in such a way that the following first-order condition characterizes the agent's choice for all $\theta$ :

$$
T^{\prime}(q(\theta))=\theta C^{\prime}(2 q(\theta))
$$

By specifying an adverse selection problem around any value of $\theta$, the slope of the equilibrium schedule in the neighborhood of this value $\theta$ is completely defined. The convexity of the equilibrium schedule at an equilibrium point is a priori not as free as under complete information. Differentiating (12) with respect to $\theta$ yields the following expression defining the second derivative of the equilibrium nonlinear price at an equilibrium point:

$$
T^{\prime \prime}(q(\theta))=\frac{C^{\prime}(2 q(\theta))}{\dot{q}(\theta)}+2 \theta C^{\prime \prime}(2 q(\theta))
$$

The convexity of the nonlinear price which describes the degree of competition between the principals is thus completely determined at any equilibrium point by how equilibrium output evolves in the neighborhood of this equilibrium point. This shows that the screening value of the contract definitively affects its commitment value.

The slope of this output schedule is itself determined by two forces playing simultaneously. First, as in standard one-principal-agent models, each principal wants to reduce the amount of good he requests from the agent in order to limit the latter's information rent. This incentive distortion depends on the distribution of the agent's types through its hazard rate. Second, as under complete information, principal 1 has also strategic incentives to increase his profit on the final market by strategically employing the agent against principal 2 .

As a result of the principals' incentives to reduce the production they request from the common agent for informational reasons, the equilibrium set is now strictly within two boundaries $\tilde{q}^{c}(\theta)$ and $q^{b}(\theta)$. The lowest bound $\tilde{q}^{c}(\theta)$ is a natural extension of the Cournot outcome under asymmetric information, had costs been replaced by virtual costs. Note that it is only at $\underline{\theta}$ that the equilibrium outputs correspond to the whole set between the competitive outcome and modified Cournot. It is thus legitimate to say that adverse selection significantly reduces the equilibrium set for other values of $\theta$. 
Proposition 7 : The output schedules $q(\theta)$ implemented in any symmetric differentiable equilibrium of the intrinsic common agency game are such that

$$
\tilde{q}^{c}(\theta)<q(\theta)<q^{b}(\theta)
$$

$\forall \theta \in\left(\underline{\theta}, \theta_{0}\right]$. In particular, $q(\theta)$ remains positive for all values of $\theta$.

Note that as $\theta_{0}$ increases (i.e., more heterogeneous agents are served), $q^{b}(\theta)$ converges to $\tilde{q}^{c}(\theta)$, so $q(\theta)$ is more tightly bounded by the presences of incomplete information.

In the case of delegated agency, it is immediate to see that $q(\theta)$ again emerges as the equilibrium allocation function, but on a possibly different participation set (i.e., for a possibly different $\theta_{0}$ ). Elsewhere in Martimort and Stole (2003), we confirm that this equality is true in general, and that (weakly) more participation occurs under delegated agency than intrinsic agency. Hence, $\theta_{0}^{D} \geq \theta_{0}^{I}$. Hence, our focus on intrinsic agency is not without much economic consequence for the present purposes of this paper.

\section{A review of the literature}

Two different kinds of externalities are at play simultaneously in the common agency models we have analyzed. First, because of decreasing returns to scale in the common manufacturer's cost function, each principal's action (the output he requests from the manufacturer) affects the agent's profit. There are thus indirect contractual externalities as in Stole (1991), Martimort (1992 and 1996) and Mezzetti (1997). Second, and contrary to this earlier common agency literature, each principal's action also affects the other principal's utility function since both retailers compete for final good on the same market. Hence, there are now also direct contractual externalities between the principals. We have shown that indirect externalities are enough to create a role for out-of-equilibrium messages even under complete information and to justify a failure of the standard version of the Revelation Principle where the agent is asked to report his type only.

Our study of nonlinear pricing games is actually more general than it first appears, and it has something to say about equilibria in large communication-mechanism common agency games. 
To see this, we can use a variation of the Taxation Principle, following the approach in Martimort and Stole (2002). ${ }^{14}$ There, we demonstrate that although the Revelation Principle cannot easily be applied to common agency environments,${ }^{15}$ it is not difficult to use an extension of the Taxation Principle from agency theory - what we call the Delegation Principle - to characterize the set of equilibria from all message games. Specifically, any equilibrium in a any commonagency communication-mechanism game is also an equilibrium to a game in which each principal chooses to offer to the agent a menu of distributions of allocations limitations) and the agent chooses an element from each principal's offered menu. ${ }^{16}$

The fact that each principal can only observe and contract upon the agent's action that he directly cares about distinguishes our paper from other common agency games under complete information like Bernheim and Whinston (1986b), Grossman and Helpman (1994), and Laussel and Le Breton (2001). In those papers, each principal can contract on the whole array of actions of the agent. This assumption implicitly requires that all those actions are observable by each principal. In the retailers-manufacturer example of this paper, this would mean that every quantity sold by a rival can be observed by a given principal: a strong assumption. ${ }^{17}$ Instead of being substitutes, the contractual activities of the different principals are thus perfect complements. It is well-known from this literature that, under complete information, the socalled truthful equilibrium implements the output which maximizes the aggregate payoff of the grand-coalition made of the retailers and the manufacturer, i.e., the monopoly outcome. The fact that the equilibrium set we found is far away from this outcome measures the consequence of imperfect bilateral contracting based on individual productions.

The modeling environment that we have considered can be viewed as complementary of

\footnotetext{
${ }^{14}$ A similar idea is independently developed in Peters (2001).

${ }^{15}$ It would require a universal message space along the lines developed in Epstein and Peters (1999).

${ }^{16}$ The main restriction in our analysis over general communication mechanism games, therefore, is to limit the principals to offering menus with only deterministic outcomes (i.e., nonlinear price-quantity schedules) rather than allowing for more general menus of distributions (i.e., nonlinear price-quantity lottery schedules). We are not aware of any equilibrium generated by lotteries that is not also generated by nonlinear prices, but at present we cannot state that this restriction is without loss of generality. A secondary restriction is that the nonlinear pricing equilibrium gives rise to smooth quantity allocations as a function of type. Given that monotonicity of outputs is a typical requirement of incentive compatibility, and that such monotonicity implies that nonlinear pricing schedules are almost everywhere smooth, this restriction is not overly strong in our opinion.

${ }^{17}$ One could argue that the observation of price contains the necessary information to infer the production of other retailer when there are only two of those. First, as we argued above, resale price maintenance contracts may be forbidden for anti-trust reasons. Second, price does not convey enough information when there are more than two retailers competing.
} 
standard models in the vertical contracting literature (see Hart and Tirole (1990), McAfee and Schwartz (1994) and Segal (1999) among others). Contrary to most of this literature, ${ }^{18}$ we give all bargaining power to retailers in designing contracts with their common manufacturer. The two game forms compare as follows. With the agent having all bargaining power, the manufacturer cannot credibly commit to the monopoly trades which would maximize the aggregate payoff of the overall coalition if offers made to each retailer are private. In equilibrium, each principal must form beliefs about what offers are made to his rivals when he contemplates an agent's deviation away from the equilibrium trade. This leeway in specifying beliefs plays the same role as the extension of the nonlinear prices that we have analyzed in our framework. It allows to generate many more outcomes than what can be achieved when beliefs are passive. In the latter case, the Cournot outcome can still be implemented just as what can be done in our model with no out-of-equilibrium extension. In a recent paper, Segal and Whinston (2001) have even shown that the equilibrium sets of both games coincide. Looking for robust predictions on the equilibrium sets, they also show that any outcome between Cournot and competitive can be sustained independently of the allocation of bargaining power between the principals and the agent. As in our paper, the finding of these equilibria relies heavily on the fact that principals offer menus with out-of-equilibrium options. Contrary to us, Segal and Whinston use discontinuous nonlinear prices to sustain equilibrium outcomes whereas, both under intrinsic and delegated common agency, we show that continuity may be kept.

Our findings on the multiplicity of equilibria of our common agency game are also clearly related to the literature on delegation in competing principal-agent pairs with publicly observable contracts analyzed in Katz (1987), Ferschtman, Judd and Kalai (1991), Caillaud, Jullien and Picard (1995) and Kühn (1997) among many others. This literature also shows that a large set of equilibria can be sustained when competing principals try to influence the behavior of their respective agents before agents play a game on their behalf. In common agency games, the interaction between the principals' contracts comes directly from the fact that the common agent's utility function depends on both contractual activities.

Also, the fact that head-to-head competition in the delegated common agency game can be

\footnotetext{
${ }^{18}$ An important exception is Segal and Whinston (2001).
} 
avoided with extended nonlinear prices is related to Wilson (1979) and Anton and Yao (1989) findings that bidders in multi-unit auctions can use their bidding strategies to achieve some relatively collusive outcomes.

In their study of equilibrium in supply schedules, Klemperer and Meyer (1989) have used ex ante uncertainty as a way to select among the many equilibria of the game under complete information. This bears some resemblance with our analysis and in particular with our desire to make credible any contractual option as being an equilibrium option for some types of the agent. With supply functions, there is a uniform price clearing the market whereas, in our model, different quantities are bought by a principal at different prices. Second, we assume adverse selection instead of ex ante uncertainty highlighting the dual role of a schedule as giving strategic advantages and reducing information rents.

\section{Conclusion}

This paper has contributed to the analysis of common agency games by stressing the role of out-of-equilibrium messages in changing the degree of competition between rival principals.

First, we have characterized the set of equilibria outcomes of an intrinsic common agency game under direct externalities between the principals and complete information. Any outcome between the Cournot and the competitive outcomes can be realized as an equilibrium outcome with nonlinear prices conveniently extended out of the equilibrium output. ${ }^{19}$ Nonlinear prices have thus a commitment value because they affect the degree of competition between principals.

Second, we have shown that the equilibrium outputs of the intrinsic common agency game remain equilibrium outcomes under delegated common agency, i.e., when the agent may refuse one of the principals' contracts. The distribution of surplus is however different than under intrinsic common agency. The role of out-of-equilibrium messages is then to insure that the competition between the principals is soft enough so that the head-to-head competition which takes place with direct mechanisms is replaced by more collusive outcomes.

\footnotetext{
${ }^{19}$ In an earlier version of this paper, we showed that, for complements, the equilibrium set corresponds to the whole set of outputs between the monopoly and the Cournot outcomes.
} 
Both under intrinsic and delegated common agency, the source of the multiplicity of equilibria is the principals' desire to manipulate the agent's behavior even out of the equilibrium. These manipulations can only be achieved with indirect mechanisms stipulating transfers for out-ofequilibrium output choices. As a consequence, direct revelation mechanisms fail to replicate the equilibrium outcomes achieved with indirect mechanisms: a failure of the Revelation Principle in common agency games.

Finally, under intrinsic common agency, we have introduced asymmetric information in order to refine within the set of these possible equilibria and characterized those equilibria. The shape of the nonlinear price is now constrained by incentive compatibility. This does somewhat restrict the set of symmetric equilibrium outcomes. The screening role of the contract somewhat undermines its commitment value. Of course, a relevant extension would be to analyze whether delegated and intrinsic common agency games have the same equilibrium sets under asymmetric information. This is a complex issue that we analyze in a companion paper, Martimort and Stole (2003). 


\section{References}

Anton, J. and D. Yao, 1989, "Split Awards, Procurement, and Innovation," Rand Journal of Economics, 20: 538-552.

Bernheim, B. D. and M. Whinston, 1986a, "Common Agency," Econometrica, 54: 923-42.

Bernheim, D. and M. Whinston, 1986b, "Menu Auctions, Resource Allocations, and Economic Influence," Quarterly Journal of Economics, 101: 1-31.

Caillaud, B., B. Jullien and P. Picard, 1995, "Competing Vertical Structures: Precommitment and Renegotiation," Econometrica, 63: 621-646.

Epstein, L. and M. Peters, 1999, "A Revelation Principle for Competing Mechanisms," Journal of Economic Theory, 88: 119-160.

Ferschtman, C., K. Judd, and E. Kalai, 1991, "Observable Contracts: Strategic Delegation and Cooperation." International Economic Review, 32: 551-559.

Grossman, G. and E. Helpman, 1994, "Protection for Sale," American Economic Review, 84: $833-850$.

Hart, O. and J. Tirole, 1990, "Vertical Integration and Market Foreclosure," Brookings Papers on Economics Activity, 205-276.

Katz, M., 1987, "Game Playing Agents: Unobservable Contracts as Precommitments," mimeo, University of California at Berkeley.

Klemperer, P. and M. Meyer, 1989, "Supply Function Equilibria in Oligopoly under Uncertainty," Econometrica, 57: 1243-1278.

Kühn, K-U., 1997, "Vertical Restraints in a Manufacturer Duopoly: The Case of Nonlinear Pricing," Rand Journal of Economics, 28: 37-62.

Laffont, J.J. and D. Martimort, 1997, "The Firm as a Multicontract Organization," Journal of Economics and Management Strategy, 6: 201-234. 
Laussel, D. and M. Le Breton, 2001, "Conflict and Cooperation: The Structure of Equilibrium Payoffs in Common Agency," Journal of Economic Theory, 100: 93-128.

McAfee, P. and M. Schwartz, 1993, "Opportunism in Multilateral Vertical Contracting: Nondiscrimination, Exclusivity and Uniformity," American Economic Review, 84: 210230.

Martimort, D., 1992, "Multi-Principaux avec Anti-Selection," Annales d'Economie et de Statistiques, 28: 1-38.

Martimort, D., 1996, "Exclusive Dealing, Common Agency, and Multiprincipals Incentive Theory," RAND Journal of Economics, 27: 1-31.

Martimort, D. and L. Stole, 1998, "Communication Spaces, Equilibria Sets and the Revelation Principle under Common Agency," mimeo, University of Chicago.

Martimort, D. and L. Stole, 2002, "The Revelation and Delegation Principles in Common Agency Games," Econometrica, 70: 1659-1673.

Martimort, D. and L. Stole, 2003, "Market Participation under Delegated and Intrinsic Common Agency Games," mimeo, January 2003.

Mezzetti, C., 1997, "Common Agency with Horizontally Differentiated Principals," RAND Journal of Economics, 28: 323-345.

Peck, J., 1996, "Competing Mechanisms and the Revelation Principle," Ohio State University, mimeo, July 1996.

Peters, M., 2001, "Common Agency and the Revelation Principle", Econometrica, 69: 13491372.

Riley, J., 1979, "Informational Equilibria," Econometrica, 47(2): 331-59.

Segal, I., 1999, "Contracting with Externalities," Quarterly Journal of Economics, 114: 337389. 
Segal, I. and M. Whinston, 2001, "Robust Predictions for Bilateral Contracting with Externalities," mimeo Stanford University, October 2001.

Stole, L., 1991, "Mechanism Design under Common Agency," chapter 1, PhD, MIT.

Tirole, J., 1988, The Theory of Industrial Organization, MIT Press.

Wilson, R, 1979, "Auctions of Shares," Quarterly Journal of Economics, 93: 675-699. 


\section{Appendix}

\section{Proof of Proposition 1 and Corollary 1:}

We take as given the nonlinear price $T_{2}\left(q_{2} \mid \theta\right)$ offered by principal 2 to the common agent and suppose that $T_{2}\left(q_{2} \mid \theta\right)-C\left(q_{1}+q_{2}\right)$ is sufficiently concave w.r.t. $q_{2}$ so that there exists a unique maximizer defined by the first-order condition, (1). In any differentiable equilibrium, the firstorder condition is necessary; we check below that the agent's objective function is sufficiently regular to guarantee that this condition is also sufficient. Define

$$
q_{2}^{*}\left(q_{1}, \theta\right) \equiv \arg \max _{q_{2}} T_{2}\left(q_{2} \mid \theta\right)-C\left(q_{1}+q_{2}, \theta\right)
$$

Under complete information, principal 1's problem is thus:

$$
\begin{gathered}
\max _{\left\{t_{1}, q_{1}\right\}} P\left(q_{1}+q_{2}^{*}\left(q_{1}, \theta\right)\right) q_{1}-t_{1} \\
\text { subject to } \\
t_{1}+T_{2}\left(q_{2}^{*}\left(q_{1}, \theta\right) \mid \theta\right)-\theta C\left(q_{1}+q_{2}^{*}\left(q_{1}, \theta\right)\right) \geq 0 .
\end{gathered}
$$

In principal 1's problem, (14) is binding because the principal wants to reduce the transfer he offers to the agent as much as possible. Substituting the value of $t_{1}$ from this binding constraint into the maximand, the principal's objective function can be written as a function, $V\left(q_{1}, \theta\right)$, of $q_{1}$ and $\theta$. Optimizing w.r.t. $q_{1}$ (and applying the envelope theorem to compute $T_{2}^{\prime}\left(q_{2}^{*}\left(q_{1}, \theta\right) \mid \theta\right)$ ) yields

$$
\begin{aligned}
& \frac{\partial}{\partial q_{1}} V\left(q_{1}, \theta\right)= \\
& \quad q_{1} P^{\prime}\left(q_{1}+q_{2}^{*}\left(q_{1}, \theta\right)\right)\left(1+\frac{\partial q_{2}^{*}\left(q_{1}, \theta\right)}{\partial q_{1}}\right)+P\left(q_{1}+q_{2}^{*}\left(q_{1}, \theta\right)\right)-\theta C^{\prime}\left(q_{1}+q_{2}^{*}\left(q_{1}, \theta\right)\right)=0 .
\end{aligned}
$$

To simplify this expression, we next calculate the value of $\frac{\partial q_{2}^{*}}{\partial q_{1}}\left(q_{1}, \theta\right)$. A necessary condition for the optimality of the agent's problem is the first-order condition (1). We will show that ex post the agent's objective is globally concave for the equilibrium nonlinear price that we construct. Hence, this condition is also sufficient. Differentiating (1) w.r.t. $q_{1}$ yields the following relationship, true for all $\left(q_{1}, \theta\right)$ :

$$
\left(T_{2}^{\prime \prime}\left(q_{2}^{*}\left(q_{1}, \theta\right) \mid \theta\right)-\theta C^{\prime \prime}\left(q_{1}+q_{2}^{*}\left(q_{1}, \theta\right)\right) \frac{\partial q_{2}^{*}\left(q_{1}, \theta\right)}{\partial q_{1}}=\theta C^{\prime \prime}\left(q_{1}+q_{2}^{*}\left(q_{1}, \theta\right)\right) .\right.
$$


From (1) and (16), the RHS of (15) above can be simplified, and for a symmetric equilibrium such that $q_{1}(\theta)=q_{2}(\theta)=q(\theta)$ and $T_{1}(q \mid \theta)=T_{2}(q \mid \theta)=T(q \mid \theta)$, we obtain our key equation for characterizing the equilibrium allocation:

$$
q(\theta) P^{\prime}(2 q(\theta))+P(2 q(\theta))=\theta C^{\prime}(2 q(\theta))-\frac{q(\theta) P^{\prime}(2 q(\theta)) \theta C^{\prime \prime}(2 q(\theta)}{T^{\prime \prime}(q(\theta) \mid \theta)-\theta C^{\prime \prime}(2 q(\theta)} .
$$

- Necessary conditions for a symmetric, differentiable equilibrium. In any differentiable equilibrium, it is necessary that the agent's objective function is locally concave at the equilibrium choice. This imposes two conditions on the Hessian of this symmetric problem computed at the equilibrium value $(q(\theta), q(\theta))$ :

$$
T^{\prime \prime}(q(\theta) \mid \theta)-\theta C^{\prime \prime}(2 q(\theta)) \leq 0 \text { for all } \theta \text {. }
$$

and

$$
\left(T^{\prime \prime}(q(\theta) \mid \theta)-\theta C^{\prime \prime}(2 q(\theta))\right)^{2} \geq\left(\theta C^{\prime \prime}(2 q(\theta))\right)^{2} \text { for all } \theta \text {. }
$$

We use these necessary local concavity conditions to derive the boundaries of the equilibrium sets. Substituting the condition (18) into (17) and noting that $C^{\prime \prime}>0$ :

$$
q(\theta) P^{\prime}(2 q(\theta))+P(2 q(\theta)) \leq \theta C^{\prime}(2 q(\theta)) .
$$

From $C(\cdot)$ being convex, $P^{\prime}(\cdot)<0$ and $P^{\prime \prime}(\cdot) \leq 0,(20)$ implies that $q(\theta) \geq q^{c}(\theta)$. Inserting (19) into (17) yields:

$$
P(2 q(\theta)) \geq \theta C^{\prime}(2 q(\theta))
$$

From $C(\cdot)$ being convex, $P^{\prime}(\cdot)<0$ and $P^{\prime \prime}(\cdot) \leq 0,(21)$ implies that $q(\theta) \leq q^{b}(\theta)$. Hence, only outputs $q(\theta) \in\left[q^{c}(\theta), q^{b}(\theta)\right]$ can be part of an equilibrium.

- Sufficient conditions for a symmetric, differentiable equilibrium. For any fixed $\theta$ and $q(\theta) \in$ $\left[q^{c}(\theta), q^{b}(\theta)\right]$ we construct quadratic transfer schedules $T(q \mid \theta)=\alpha+\beta q+\frac{1}{2} \gamma q^{2}$ that form an equilibrium and generate $q(\theta)$ as a symmetric equilibrium output.

Assuming that the principals' and the agent's programs are each concave, $q(\theta)$ will be chosen by the agent if and only if the agent's first-order condition is satisfied:

$$
\beta+\gamma q(\theta)=\theta C^{\prime}(2 q(\theta))
$$


The posited transfer function will be chosen by each principal if and only if each principal's first-order condition (17) for $q$ is satisfied

$$
q(\theta) P^{\prime}(2 q(\theta))+P(2 q(\theta))=\theta C^{\prime}(2 q(\theta))-\frac{q(\theta) P^{\prime}(2 q(\theta)) \theta C^{\prime \prime}(2 q(\theta)}{\gamma-\theta C^{\prime \prime}(2 q(\theta)},
$$

and the agent earns zero rents:

$$
2\left(\alpha+\beta q(\theta)+\frac{1}{2} \gamma q(\theta)^{2}\right)=\theta C(2 q(\theta)) .
$$

The above three equations can be solved uniquely for the equilibrium values of $\{\alpha, \beta, \gamma\}$.

$$
\begin{gathered}
\alpha=\frac{\theta}{2}\left(C(2 q(\theta))+q(\theta)\left(-2 C^{\prime}(2 q(\theta))+\frac{q(\theta)\left(P(2 q(\theta))-\theta C^{\prime}(2 q(\theta))\right) C^{\prime \prime}(2 q(\theta))}{P(2 q(\theta))-\theta C^{\prime}(2 q(\theta))+q(\theta) P^{\prime}(2 q(\theta))}\right)\right), \\
\beta=\theta\left(C^{\prime}(2 q(\theta))+\frac{q(\theta)\left(-P(2 q(\theta))+\theta C^{\prime}(2 q(\theta))\right) C^{\prime \prime}(2 q(\theta))}{P(2 q(\theta))-\theta C^{\prime}(2 q(\theta))+q(\theta) P^{\prime}(2 q(\theta))}\right) \\
\gamma=\frac{\theta\left(P(2 q(\theta))-\theta C^{\prime}(2 q(\theta))\right) C^{\prime \prime}(2 q(\theta))}{P(2 q(\theta))-\theta C^{\prime}(2 q(\theta))+q(\theta) P^{\prime}(2 q(\theta))} .
\end{gathered}
$$

These values will comprise an equilibrium if both the agent's program and the principals' programs are concave.

Concavity of agent's program: Global concavity of the agent's problem requires that

$$
\gamma-\theta C^{\prime \prime}\left(q_{1}+q_{2}\right) \leq 0 \text { for all }\left(q_{1}, q_{2}\right)
$$

and

$$
\left(\gamma-\theta C^{\prime \prime}\left(q_{1}+q_{2}\right)\right)\left(\gamma-\theta C^{\prime \prime}\left(q_{1}+q_{2}\right)\right) \geq \theta^{2}\left(C^{\prime \prime}\left(q_{1}+q_{2}\right)\right)^{2} \text { for all }\left(q_{1}, q_{2}\right)
$$

These conditions are jointly satisfied if and only if $T^{\prime \prime}(q(\theta) \mid \theta)=\gamma \leq 0$. Hence, providing that $\gamma \leq 0$, the agent's program is globally concave for any $(\alpha, \beta)$. Note that the above value of $\gamma$ is non positive if $q(\theta) \in\left[q^{c}(\theta), q^{b}(\theta)\right]$, so the agent's program is concave for the constructed equilibrium tariff schedule.

Concavity of principals' programs. Given that $\frac{\partial q_{2}^{*}\left(q_{1}, \theta\right)}{\partial q_{1}}=\frac{\theta C^{\prime \prime}\left(q_{1}+q_{2}^{*}\left(q_{1}, \theta\right)\right)}{\gamma-\theta C^{\prime \prime}\left(q_{1}+q_{2}^{*}\left(q_{1}, \theta\right)\right)}$, we obtain:

$$
\begin{aligned}
& \frac{\partial^{2} V}{\partial q_{1}^{2}}\left(q_{1}, \theta\right)= \\
& \quad q_{1} P^{\prime \prime}\left(q_{1}+q_{2}^{*}\left(q_{1}, \theta\right)\right) \frac{\gamma^{2}}{\left(\gamma-\theta C^{\prime \prime}\left(q_{1}+q_{2}^{*}\left(q_{1}, \theta\right)\right)\right)^{2}}+2 P^{\prime}\left(q_{1}+q_{2}^{*}\left(q_{1}, \theta\right)\right)\left(\frac{\gamma}{\gamma-\theta C^{\prime \prime}\left(q_{1}+q_{2}^{*}\left(q_{1}, \theta\right)\right)}\right) \\
& \quad+q_{1} P^{\prime}\left(q_{1}+q_{2}^{*}\left(q_{1}, \theta\right)\right)\left(\frac{\gamma \theta C^{\prime \prime \prime}\left(q_{1}+q_{2}^{*}\left(q_{1}, \theta\right)\right)}{\left(\gamma-\theta C^{\prime \prime}\left(q_{1}+q_{2}^{*}\left(q_{1}, \theta\right)\right)\right)^{3}}\right)-\theta C^{\prime \prime}\left(q_{1}+q_{2}^{*}\left(q_{1}, \theta\right)\right)\left(\frac{\gamma}{\gamma-\theta C^{\prime \prime}\left(q_{1}+q_{2}^{*}\left(q_{1}, \theta\right)\right)}\right) .
\end{aligned}
$$


The first term is negative thanks to $P^{\prime \prime}(\cdot) \leq 0$. The third term is negative since $\gamma \leq 0$ and $C^{\prime \prime \prime} \geq 0$. The second term is negative since $\frac{\gamma}{\gamma-\theta C^{\prime \prime}\left(q_{1}+q_{2}^{*}\left(q_{1}, \theta\right)\right)}>0$ and $P^{\prime}(\cdot)<0$. Taking into account that $C^{\prime \prime} \geq 0$, the fourth term is also negative. Hence, each principal's program is strictly concave and so the posited quadratic tariffs form a Nash equilibrium and implement $q(\theta)$.

Proof of Proposition 2: Assume that principal 2 offers the direct revelation mechanism $\left\{t_{2}(\theta), q_{2}(\theta)\right\}$, then principal 1's problem is:

$$
\begin{array}{cc}
\max _{\left\{t_{1}, q_{1}\right\}} & P\left(q_{1}+q_{2}(\theta)\right) q_{1}-t_{1}, \\
& \text { subject to } \\
t_{1}+t_{2}(\theta)- & \theta C\left(q_{1}+q_{2}(\theta)\right) \geq 0 .
\end{array}
$$

Again (24) is binding at the optimum of principal 1's problem. Inserting the corresponding value of $t_{1}$ into principal 1's objective function, observing that the corresponding maximand is concave in $q_{1}$ and optimizing with respect to $q_{1}$, yields:

$$
q_{1} P^{\prime}\left(q_{1}+q_{2}(\theta)\right)+P\left(q_{1}+q_{2}(\theta)\right)=\theta C^{\prime}\left(q_{1}+q_{2}(\theta)\right)
$$

In a symmetric equilibrium, we obtain $q_{1}(\theta)=q_{2}(\theta)=q^{c}(\theta)$.

Proof of Proposition 3: The proof starts as in the proof of Proposition 1. (15) must be replaced by

$\frac{\partial V}{\partial q_{1}}\left(q_{1}, \theta\right)=q_{1} P^{\prime}\left(q_{1}\right)+P\left(q_{1}\right)-\theta C^{\prime}\left(q_{1}+q_{2}^{*}\left(q_{1}, \theta\right)\right)+\left[T_{2}^{\prime}\left(q_{2}^{*}\left(q_{1}, \theta\right) \mid \theta\right)-C^{\prime}\left(q_{1}+q_{2}^{*}\left(q_{1}, \theta\right)\right)\right] \frac{\partial q_{2}^{*}\left(q_{1}, \theta\right)}{\partial q_{1}}=0$.

The first-order condition $T_{2}^{\prime}\left(q_{2}^{*}\left(q_{1}, \theta\right) \mid \theta\right)-\theta C^{\prime}\left(q_{1}+q_{2}^{*}\left(q_{1}, \theta\right)\right)=0$ characterizes the agent's choice of output. In a symmetric equilibrium, conjectures must be correct and thus $q_{2}^{*}(q, \theta)=q(\theta)=$ $\tilde{q}^{m}(\theta)$. It is routine to check that the same outcome can be implemented with direct mechanisms. 


\section{Proof of Proposition 4 :}

- Nonexistence of pure-strategy equilibria with each principal served.

Suppose that a pure-strategy symmetric equilibrium exists with direct mechanisms and that it sustains a common agency outcome where the agent contracts simultaneously with both principals. Let $(t(\theta), q(\theta))$ denote the associated equilibrium transfer and output. In such an equilibrium, principals decrease as much as possible the transfers they offer to the agent so that the agent's equilibrium rent satisfies:

$$
U(\theta)=2 t(\theta)-\theta C(2 q(\theta))=\max \{0, t(\theta)-\theta C(q(\theta))\}
$$

where the outside option of the agent on the right-hand side allows the agent to choose to contract with one principal or none of them, depending upon which produces greater payoffs.

It follows that the greater outside payoff is obtained when the agent refuses only one contract. To see this, note that if it were not the case, we would have $2 t(\theta)-\theta C(2 q(\theta))=0$ or $t(\theta)=$ $\theta C(2 q(\theta)) / 2>\theta C(q(\theta))$, where the latter inequality follows from the convexity of costs. Hence, $t(\theta)-\theta C(q(\theta))>0$ and the relevant outside option is serving one principal. Using this result, we get

$$
t(\theta)=\theta(C(2 q(\theta))-C(q(\theta))) .
$$

Consider now an upward deviation in output by principal 1, that is $q_{1}>q(\theta)$ and the associated transfer

$$
t_{1}=\theta\left(C\left(q_{1}+q(\theta)\right)-C(q(\theta))\right) .
$$

Then, from the above definition of $t(\theta)$, we have

$$
t(\theta)-\theta C\left(q_{1}+q(\theta)\right)<-\theta C\left(q_{1}\right),
$$

so

$$
t_{1}+t(\theta)-\theta C\left(q_{1}+q(\theta)\right)<t_{1}-\theta C\left(q_{1}\right)
$$

and the agent prefers not to take principal 2's contract given principal 1's deviation. The profit of the deviating principal who chooses $q_{1}>q(\theta)$ is:

$$
V(q(\theta), \theta)=q_{1} P\left(q_{1}\right)-\theta\left(C\left(q_{1}+q(\theta)\right)-C(q(\theta))\right) .
$$


The profit along the equilibrium path, however, is thus:

$$
V\left(q_{1}, \theta\right)=q(\theta) P(2 q(\theta))-\theta(C(2 q(\theta))-C(q(\theta))) .
$$

The cost components of the principal's payoffs are continuous as $q_{1}$ increases above $q(\theta)$, but the revenues discontinuously jump upwards as $q_{1}$ increases above $q(\theta)$ if $q(\theta)>0$. Hence, the principal's profits jump upwards for a slight deviation upward from the conjectured equilibrium output, $q(\theta)$. We conclude that there does not exist a pure-strategy equilibrium in which both principals contract with the agent.

- Existence of pure-strategy equilibria in which each principal served is exclusively served with equal probability.

Consider now that the possibility of a pure-strategy equilibrium in which both principals compete head-to-head for the exclusive service of the agent. A necessary condition for equilibrium is that the principals bid away all of their profit from being exclusive and each offers a direct revelation mechanism with associated transfer $t(\theta)=2 q^{m}(\theta) P\left(2 q^{m}(\theta)\right)$ and quantity $2 q^{m}(\theta)$. Such an offer is the most attractive that a principal can make. Moreover, with these offers, the agent is willing to randomize (equally in a symmetric equilibrium) between the principals, providing that the agent earns more rents than when contracting with both principals simultaneously. This provision is satisfied if and only if

$$
4 q^{m}(\theta) P\left(2 q^{m}(\theta)\right)-\theta C\left(4 q^{m}(\theta)\right)<2 q^{m}(\theta) P\left(2 q^{m}(\theta)\right)-\theta C\left(2 q^{m}(\theta)\right)
$$

or

$$
2 q^{m}(\theta) P\left(2 q^{m}(\theta)\right)-\theta\left(C\left(4 q^{m}(\theta)\right)-C\left(2 q^{m}(\theta)\right)\right)<0,
$$

which is condition (5) of the proposition. Note that because we have assumed that the monopoly outcome yields a positive profit

$$
2 q^{m}(\theta) P\left(2 q^{m}(\theta)\right)-\theta C\left(2 q^{m}(\theta)\right)>0
$$

and since $C\left(4 q^{m}(\theta)\right)-2 C\left(2 q^{m}(\theta)\right)>0$ from $C(\cdot)$ convexity, the two inequalities (28) and (29) are not mutually exclusive. 


\section{Proof of Proposition 5:}

Let us fix $q(\theta) \in\left[q^{c}(\theta), q^{b}(\theta)\right]$ and let us construct the equilibrium schedule $T_{d}(\cdot \mid \theta)$ that sustains this output in a symmetric equilibrium under common agency.

- Upwards deviations by principal 1 and extension of $T_{d}(\cdot \mid \theta)$ below the equilibrium output: We first construct the equilibrium schedule $T_{d}(\cdot \mid \theta)$ under delegated common agency so that principal 1 does not want to induce more production from the agent. For simplicity, we denote by $T_{d}(\theta)=T_{d}(q(\theta) \mid \theta)$ the equilibrium value of the transfer.

Let also denote by $\Pi(\theta)$ the principal's equilibrium payoff and by $U(\theta)$ the agent's equilibrium rent (which must be positive since the agent has always the option of refusing both contracts). This rent is constructed below so that the agent is indifferent between taking both contracts and that offered by principal 2 only.

By definition, we thus have:

$$
q(\theta) P(2 q(\theta))+T_{d}(\theta)-\theta C(2 q(\theta))=\Pi(\theta)+U(\theta) .
$$

Let us construct the extension of $T_{d}(\cdot \mid \theta)$ for $q \in[0, q(\theta)]$ in such a way that principal 1 gets a constant payoff if he deviates upwards and offers $q_{1} \geq q(\theta)$ for $q_{1}$ not too large so that $q_{2}^{*}\left(q_{1}, \theta\right)$ remains positive (this upper bound is made precise below). The payoff of the deviation is

$$
V\left(q_{1}, \theta\right)=P\left(q_{1}+q_{2}^{*}\left(q_{1}, \theta\right)\right) q_{1}+T_{d}\left(q_{2}^{*}\left(q_{1}, \theta\right) \mid \theta\right)-\theta C\left(q_{1}+q_{2}^{*}\left(q_{1}, \theta\right)\right)-U(\theta)
$$

where $q(\theta) \geq q_{2}^{*}\left(q_{1}, \theta\right) \geq 0$ for such a $q_{1}$. It is constant for all $q_{1}$ greater than $q(\theta)$ if $\frac{\partial V}{\partial q_{1}}=0$. This writes as (15) and taking into account (1) which characterizes the agent's behavior for $q_{1} \geq q(\theta)$, we obtain the following differential equation mapping any $q_{1}$ into the agent's best response $q_{2}^{*}\left(q_{1}, \theta\right)$ which ensures that principal 1 does not want to deviate:

$$
\frac{\partial q_{2}^{*}\left(q_{1}, \theta\right)}{\partial q_{1}}=-\frac{q_{1} P^{\prime}\left(q_{1}+q_{2}^{*}\left(q_{1}, \theta\right)\right)+P\left(q_{1}+q_{2}^{*}\left(q_{1}, \theta\right)\right)-\theta C^{\prime}\left(q_{1}+q_{2}^{*}\left(q_{1}, \theta\right)\right)}{q_{1} P^{\prime}\left(q_{1}+q_{2}^{*}\left(q_{1}, \theta\right)\right)} .
$$

With the boundary condition $q_{2}^{*}(q(\theta), \theta)=q(\theta)$, (30) defines the out-of-equilibrium outputs below the equilibrium value which are needed to hold the pure strategy equilibrium if the principal 1 deviates. 
Lemma 2 : For all $q_{1} \in\left[q(\theta), \tilde{q}^{m}(\theta)\right]$, we have $q_{2}^{*}\left(q_{1}, \theta\right) \in\left[q^{c}\left(q_{1}, \theta\right), q^{b}\left(q_{1}, \theta\right)\right]$ where

$$
q_{1} P^{\prime}\left(q_{1}+q^{c}\left(q_{1}, \theta\right)\right)+P\left(q_{1}+q_{1}^{c}\left(q_{1}, \theta\right)\right)=\theta C^{\prime}\left(q_{1}+q^{c}\left(q_{1}, \theta\right)\right)
$$

and

$$
P\left(q_{1}+q_{1}^{b}\left(q_{1}, \theta\right)\right)=\theta C^{\prime}\left(q_{1}+q^{b}\left(q_{1}, \theta\right)\right)
$$

First, note that $q^{c}\left(q_{1}, \theta\right)$ and $q^{b}\left(q_{1}, \theta\right)$ are both decreasing functions of $q_{1}$. Moreover, by direct identification, we have $q^{b}\left(q_{1}, \theta\right)=2 q^{b}(\theta)-q_{1}$. Also, $q^{c}\left(2 q^{m}(\theta), \theta\right)=0$.

Take then a solution to (30) which starts at $q_{1}=q(\theta) \in\left[q^{c}(\theta), q^{b}(\theta)\right]$. This solution remains above $q^{c}\left(q_{1}, \theta\right)$. Suppose on the contrary that it does not and denote by $q_{1}^{\prime}$ the first value of $q_{1}$ such that $q_{2}^{*}\left(q_{1}^{\prime}, \theta\right)=q^{c}\left(q_{1}^{\prime}, \theta\right)$. At this point, we have $\frac{\partial q_{2}^{*}\left(q_{1}^{\prime}, \theta\right)}{\partial q_{1}}=0$. Since $q_{1}^{\prime}>q(\theta)$ and $\frac{\partial q^{c}\left(q_{1}^{\prime}, \theta\right)}{\partial q_{1}}<0$, we have $q_{2}^{*}\left(q_{1}^{\prime}-\epsilon, \theta\right)<q^{c}\left(q_{1}^{\prime}-\epsilon, \theta\right)$ for $\epsilon$ small enough. A contradiction.

$q_{2}^{*}\left(q_{1}, \theta\right)$ remains also below $q^{b}\left(q_{1}, \theta\right)$. Suppose on the contrary that it does not and denote by $q_{1}^{\prime \prime}$ the first value of $q_{1}$ such that $q_{2}^{*}\left(q_{1}^{\prime \prime}, \theta\right)=q^{b}\left(q_{1}^{\prime \prime}, \theta\right)$. At this point, we have $\frac{\partial q_{2}^{*}\left(q_{1}^{\prime \prime}, \theta\right)}{\partial q_{1}}=$ $\frac{\partial q^{b}\left(q_{1}^{\prime \prime}, \theta\right)}{\partial q_{1}}=-1$ and, it can be checked that this equality is true for all derivatives of those two functions. Hence, $q_{2}^{*}\left(q_{1}, \theta\right)=q^{b}\left(q_{1}, \theta\right)$ for all $q_{1}$ in a small neighborhood of $q_{1}^{\prime \prime}$. A contradiction.

As a consequence of Lemma 2 , we have $q_{2}^{*}\left(q_{1}, \theta\right)=0$ only for $q_{1} \geq \tilde{q}(\theta)$ where $\tilde{q}(\theta)>2 q^{m}(\theta)$. Note that for such $q_{1}$, we have

$$
V\left(q_{1}, \theta\right)=q_{1} P\left(q_{1}\right)+T_{d}(0 \mid \theta)-\theta C\left(q_{1}\right)-U(\theta)
$$

and that $V\left(q_{1}, \theta\right)$ is decreasing in $q_{1}$ so that those large deviations in $q_{1}$ are not beneficial for principal 1 either. Also, because $q_{2}^{*}\left(q_{1}, \theta\right)>q^{c}\left(q_{1}, \theta\right), q_{2}^{*}\left(q_{1}, \theta\right)$ is decreasing in $q_{1}$. Let denote by $\phi\left(q_{2}\right)$ its inverse function which is also differentiable, decreasing over $[0, q(\theta)]$ and such that $\phi(q(\theta))=q(\theta)$. 
Given a schedule $q_{2}^{*}\left(q_{1}, \theta\right)$ which is defined above, using (1) and the initial condition $T_{d}(0 \mid \theta)=$ 0 , simple integration leads to

$$
T_{d}(q \mid \theta)=\int_{0}^{q} \theta C^{\prime}\left(\phi\left(q_{2}\right)+q_{2}\right) d q_{2}
$$

for $q \in[0, q(\theta)]$. In particular, the equilibrium value of the transfer is

$$
T_{d}(\theta)=T_{d}(q(\theta) \mid \theta)=\int_{0}^{q(\theta)} \theta C^{\prime}\left(\phi\left(q_{2}\right)+q_{2}\right) d q_{2} .
$$

- Downwards deviations by principal 1 and extension of $T_{d}(\cdot \mid \theta)$ above the equilibrium output: Denote by $U(\theta)$ the agent's equilibrium rent in a delegated common agency game. Since, the agent takes both contracts in equilibrium, this rent is thus:

$$
U(\theta)=2 T_{d}(q(\theta) \mid \theta)-\theta C(2 q(\theta))
$$

Using (31), we obtain

$$
\begin{gathered}
U(\theta)=\theta\left(2 \int_{0}^{q(\theta)} C^{\prime}\left(\phi\left(q_{2}\right)+q_{2}\right) d q_{2}-C(2 q(\theta))\right) \\
=\theta\left(2 \int_{0}^{q(\theta)} C^{\prime}\left(\phi\left(q_{2}\right)+q_{2}\right)\left(1+\dot{\phi}\left(q_{2}\right)-\dot{\phi}\left(q_{2}\right)\right) d q_{2}-C(2 q(\theta))\right) .
\end{gathered}
$$

Integrating by parts the first term on the right-hand side and changing variables yields

$$
U(\theta)=\theta\left(C(2 q(\theta))-2 C(\tilde{q}(\theta))+\int_{q(\theta)}^{\tilde{q}(\theta)} C^{\prime}\left(q_{1}+q_{2}^{*}\left(q_{1}, \theta\right)\right) d q_{1}\right) .
$$

For $q>q(\theta)$, let us extend $T_{d}(q \mid \theta)$ linearly as

$$
T_{d}(q \mid \theta)=T_{d}(\theta)+\theta C^{\prime}\left(q^{*}\right)(q-q(\theta))
$$

for some $q^{*}$ to be defined below such that $q^{*}>q(\theta)$. Note that $T_{d}(q \mid \theta)$ is continuous at $q(\theta)$ but not necessarily continuously differentiable and indeed it won’t be (see Figure 1).

With such a definition, the agent chooses $q_{2}=q^{*}$ if he serves only principal 2. Indeed, choosing any $q_{2} \leq q(\theta)$ is dominated by choosing $q(\theta)$ This yields him a positive rent from taking only principal 2's contract given by:

$$
U_{2}(\theta)=T_{d}(\theta)+\theta\left(C^{\prime}\left(q^{*}\right)\left(q^{*}-q(\theta)\right)-C\left(q^{*}\right)\right)
$$


To be sure that principal 1 has reduced his own transfer as much as possible in equilibrium, it must be that $U(\theta)=U_{2}(\theta)$ in equilibrium. Given (33) and (31), it must be that $q^{*}>q(\theta)$ satisfies:

$$
\int_{q(\theta)}^{\tilde{q}(\theta)} C^{\prime}\left(q_{1}+q_{2}^{*}\left(q_{1}, \theta\right)\right) d q_{1}-C(\tilde{q}(\theta))=\psi\left(q^{*}\right)=C^{\prime}\left(q^{*}\right)\left(q^{*}-q(\theta)\right)-C\left(q^{*}\right) .
$$

It is easy to check that $\psi(\cdot)$ is strictly increasing. Moreover, $\psi(q(\theta))=-C(q(\theta))<\int_{q(\theta)}^{\tilde{q}(\theta)} C^{\prime}\left(q_{1}+\right.$ $\left.q_{2}^{*}\left(q_{1}, \theta\right)\right) d q_{1}-C(\tilde{q}(\theta))$ since $q_{2}^{*}\left(q_{1}, \theta\right) \geq 0$ on the relevant interval $[q(\theta), \tilde{q}(\theta)]$, and $\psi(\tilde{q}(\theta))=$ $C^{\prime}(\tilde{q}(\theta))(\tilde{q}(\theta)-q(\theta))-C(\tilde{q}(\theta))>\int_{q(\theta)}^{\tilde{q}(\theta)} C^{\prime}\left(q_{1}+q_{2}^{*}\left(q_{1}, \theta\right)\right) d q_{1}-C(\tilde{q}(\theta))$ since $q_{1}+q_{2}^{*}\left(q_{1}, \theta\right)$ increases with $q_{1}$ for $q_{1} \geq q(\theta)$ and thus $\int_{q(\theta)}^{\tilde{q}(\theta)} C^{\prime}\left(q_{1}+q_{2}^{*}\left(q_{1}, \theta\right)\right) d q_{1}<C^{\prime}(\tilde{q}(\theta))(\tilde{q}(\theta)-q(\theta))$. Hence, there exists a unique $q^{*}>q(\theta)$ such that (34) holds.

Note also that $q^{*}<2 q(\theta)$. Indeed, we have $\psi(2 q(\theta))=C^{\prime}(2 q(\theta)) q(\theta)-C(2 q(\theta))>$ $\int_{q(\theta)}^{\tilde{q}(\theta)} C^{\prime}\left(q_{1}+q_{2}^{*}\left(q_{1}, \theta\right)\right) d q_{1}-C(\tilde{q}(\theta)) \geq \int_{q(\theta)}^{\tilde{q}(\theta)}\left(1+\frac{\partial q_{2}^{*}\left(q_{1}, \theta\right)}{\partial q_{1}}\right) C^{\prime}\left(q_{1}+q_{2}^{*}\left(q_{1}, \theta\right)\right) d q_{1}-C(\tilde{q}(\theta))=$ $-C(2 q(\theta))$ because $\frac{\partial q_{2}^{*}\left(q_{1}, \theta\right)}{\partial q_{1}}<0$.

Let us now consider a deviation by principal 1 to induce less production by the agent than in the equilibrium, $q_{1}<q(\theta)$. For $q_{1} \in\left[q^{*}-q(\theta), q(\theta)\right]$, the agent chooses $q_{2}^{*}\left(q_{1}, \theta\right)=q(\theta)$ and principal 1 gets from such a deviation:

$$
V\left(q_{1}, \theta\right)=P\left(q_{1}+q(\theta)\right) q_{1}+T_{d}(\theta)-\theta C\left(q_{1}+q(\theta)\right)-U(\theta) .
$$

Since $q_{1} \leq q(\theta) \leq q^{b}(\theta)$, the best of such deviation is $q(\theta)$ itself. For $q_{1} \in\left[0, q^{*}-q(\theta)\right]$, the agent chooses $q_{2}^{*}\left(q_{1}, \theta\right)=q^{*}-q_{1}$ and principal 1 gets from such a deviation:

$$
V\left(q_{1}, \theta\right)=P\left(q^{*}\right) q_{1}+T_{d}(\theta)+\theta C^{\prime}\left(q^{*}\right)\left(q^{*}-q(\theta)\right)-\theta C\left(q^{*}\right)-U(\theta)
$$

which is increasing and maximized over $\left[0, q^{*}-q(\theta)\right]$ for $q_{1}=q^{*}-q(\theta)$. Since $V(\cdot, \theta)$ is continuous in $q_{1}$, the best downward deviation by principal 1 is $q(\theta)$ itself.

- Agent's Behavior: First, note that

$$
T_{d}\left(q_{i} \mid \theta\right)=T_{d}(\theta)+\theta C^{\prime}\left(q^{*}\right)\left(q_{i}-q(\theta) \text { for } q_{i} \geq q(\theta)\right.
$$

and

$$
T_{d}\left(q_{i} \mid \theta\right)=\int_{0}^{q_{i}} C^{\prime}(q+\phi(q)) d q \text { for } q_{i} \leq q(\theta)
$$


Let us denote the agent's payoff as

$$
J\left(q_{1}, q_{2}\right)=T_{d}\left(q_{1} \mid \theta\right)+T_{d}\left(q_{2} \mid \theta\right)-\theta C\left(q_{1}+q_{2}\right) .
$$

We want to prove that $J(\cdot)$ is maximized for $q_{1}=q_{2}=q(\theta)$. For that, we will decompose the $\left(q_{1}, q_{2}\right)$ space into several pieces.

Note first that, if $q_{i} \geq q(\theta)$ for at least one $i$, we have:

$$
\frac{\partial J\left(q_{1}, q_{2}\right)}{\partial q_{i}}=\theta\left(C^{\prime}\left(q^{*}\right)-C^{\prime}\left(q_{1}+q_{2}\right)\right) \leq 0
$$

if and only if $q_{1}+q_{2} \geq q^{*}$. On the rectangle $[0, q(\theta)] \times[q(\theta),+\infty$ (, the maximum of $J(\cdot)$ is achieved for $q_{2}=q^{*}-q_{1}$ if $q_{1} \in\left[0, q^{*}-q(\theta)\right]$ and it is worth

$$
\hat{J}\left(q_{1}\right)=T_{d}\left(q_{1} \mid \theta\right)+T_{d}(\theta)-\theta C^{\prime}\left(q^{*}\right) q_{1}-C\left(q^{*}\right)
$$

This maximum of $J(\cdot)$ is instead achieved for $q_{2}=q(\theta)$ if $q_{1} \in\left[q^{*}-q(\theta), q(\theta)\right]$.

We can proceed similarly on the rectangle $[q(\theta),+\infty(\times[0, q(\theta)]$ by permuting indices.

Finally, given that $q^{*}<2 q(\theta), J(\cdot)$ is maximum at $(q(\theta), q(\theta))$ on the rectangle $[q(\theta),+\infty(\times[q(\theta),+\infty($.

We now prove that $\hat{J}(\cdot)$ is maximum for $q_{1}=q^{*}-q(\theta)$. This will finally allow us to restrict the finding of the optimal outputs on the square $[0, q(\theta)] \times[0, q(\theta)]$. First notice that, for $q_{1} \in\left[0, q^{*}-q(\theta)\right]$

$$
\hat{J}^{\prime}\left(q_{1}\right)=\theta\left(C^{\prime}\left(q_{1}+\phi\left(q_{1}\right)\right)-C^{\prime}\left(q^{*}\right)\right) \text {. }
$$

Moreover, $\left.\hat{J}^{\prime \prime}\left(q_{1}\right)=\theta C^{\prime \prime}\left(q_{1}+\phi\left(q_{1}\right)\right)\left(1+\phi^{\prime} q_{1}\right)\right)$. Since $\frac{\partial q_{1}^{*}\left(q_{2}, \theta\right)}{\partial q_{2}}<0$ and $1+\frac{\partial q_{1}^{*}\left(q_{2}, \theta\right)}{\partial q_{2}}>0$ from (30) (where indices have been permuted), we have $\hat{J}^{\prime \prime}\left(q_{1}\right)<0$ and $\hat{J}^{\prime}\left(q_{1}\right) \geq \hat{J}^{\prime}\left(q^{*}-q(\theta)\right)$. That $q^{*}<2 q(\theta)$ and $\phi(\cdot)$ decreasing imply that $\phi\left(q^{*}-q(\theta)\right) \geq \phi(q(\theta))=q(\theta)$. Finally, we have $\hat{J}^{\prime}\left(q^{*}-q(\theta)\right) \geq \theta\left(C^{\prime}\left(q^{*}-q(\theta)+q(\theta)\right)-C^{\prime}(q(\theta))\right)=0$. Hence, $\hat{J}(\cdot)$ is increasing on $\left[0, q^{*}-q(\theta)\right]$ and thus maximized for $q_{1}=q^{*}-q(\theta)$.

Let us now consider the maximum of $J(\cdot)$ on the domain $[0, q(\theta)] \times[0, q(\theta)]$. In fact, we have:

$$
\frac{\partial J\left(q_{1}, q_{2}\right)}{\partial q_{1}}=\theta\left(C^{\prime}\left(q_{1}+\phi\left(q_{1}\right)\right)-C^{\prime}\left(q_{1}+q_{2}\right)\right) \geq \theta\left(C^{\prime}\left(q_{1}+q(\theta)\right)-C^{\prime}\left(q_{1}+q_{2}\right)\right)
$$


since $\phi\left(q_{1}\right) \geq q(\theta)$ and thus $\frac{\partial J\left(q_{1}, q_{2}\right)}{\partial q_{1}} \geq 0$ since $q(\theta) \geq q_{2}$. Similarly, $\frac{\partial J\left(q_{1}, q_{2}\right)}{\partial q_{2}} \geq 0$ and thus $J(\cdot)$ is maximized at $(q(\theta), q(\theta))$.

\section{- Schedules:}

On Figure 1 below, we have drawn the equilibrium schedule constructed under delegated common agency. It is important to notice that the curve is not differentiable everywhere and is significantly less convex than the indifference curve for low levels of outputs.

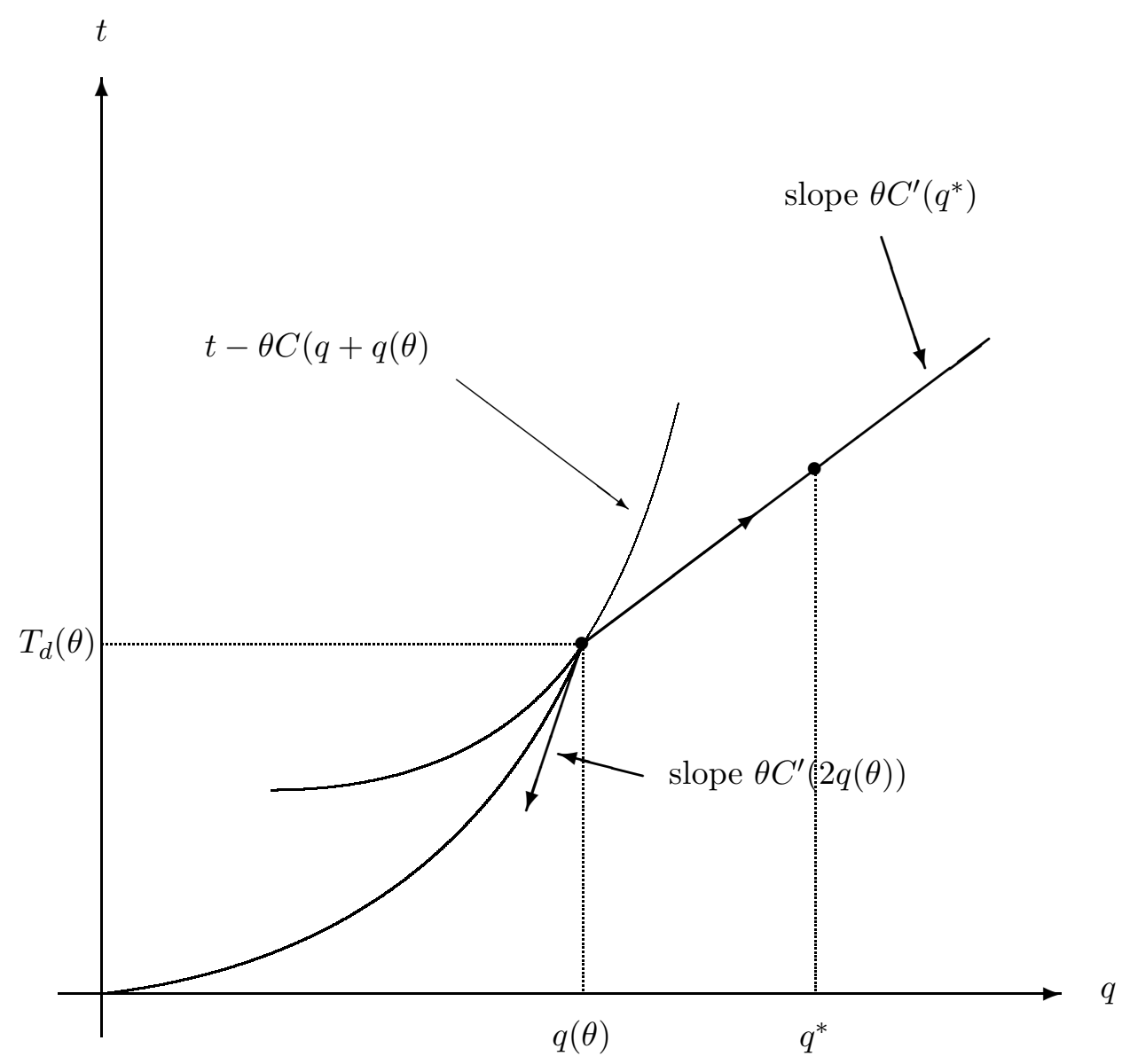

Figure 1: Nonlinear price schedule $T_{d}(\cdot \mid \theta)$.

\section{Proofs of Propositions 6 and 7:}

- (8) indicates that $U(\cdot)$ is decreasing because $u_{\theta}^{1}\left(q_{1}(\theta), \theta\right)=-C\left(q_{1}(\theta)+q_{2}^{*}\left(q_{1}(\theta), \theta\right)\right)<0$. Hence, 
the participation constraint $(10)$ is binding only at $\theta_{0}$. Henceforth:

$$
U(\theta)=\int_{\theta}^{\theta_{0}} C\left(q_{1}(z)+q_{2}^{*}\left(q_{1}(z), z\right)\right) d z .
$$

Inserting into the principal's objective function and integrating by parts yields:

$$
\int_{\underline{\theta}}^{\theta_{0}} f(\theta) U(\theta) d \theta=\int_{\underline{\theta}}^{\theta_{0}} \frac{F(\theta)}{f(\theta)} f(\theta) C\left(q_{1}(\theta)+q_{2}^{*}\left(q_{1}(\theta), \theta\right)\right) d \theta .
$$

The objective function to be optimized pointwise becomes a function of $q_{1}$ only, namely:

$$
V\left(q_{1}, \theta\right)=q_{1} P\left(q_{1}+q_{2}^{*}\left(q_{1}, \theta\right)\right)+u^{1}\left(q_{1}, \theta\right)+\frac{F(\theta)}{f(\theta)} u_{\theta}^{1}\left(q_{1}, \theta\right) .
$$

Assuming the concavity of $V(\cdot)$ with respect to $q_{1}$ and optimizing pointwise w.r.t. $q_{1}$ yields:

$$
\frac{\partial V\left(q_{1}, \theta\right)}{\partial q_{1}}=
$$

$\left(35 \not_{1} P^{\prime}\left(q_{1}+q_{2}^{*}\left(q_{1}, \theta\right)\right)\left(1+\frac{\partial q_{2}^{*}\left(q_{1}, \theta\right)}{\partial q_{1}}\right)+P\left(q_{1}+q_{2}^{*}\left(q_{1}, \theta\right)\right)+u_{1}^{1}\left(q_{1}, \theta\right)+\frac{F(\theta)}{f(\theta)} u_{1 \theta}^{1}\left(q_{1}, \theta\right)=0\right.$.

Using the Envelope Theorem:

$$
\begin{gathered}
u_{1}^{1}\left(q_{1}, \theta\right)=-\theta C^{\prime}\left(q_{1}+q_{2}^{*}\left(q_{1}(\theta), \theta\right)\right) \\
u_{1 \theta}^{1}\left(q_{1}, \theta\right)=-C^{\prime}\left(q_{1}+q_{2}^{*}\left(q_{1}, \theta\right)\right)-\theta C^{\prime \prime}\left(q_{1}+q_{2}^{*}\left(q_{1}, \theta\right)\right) \frac{\partial q_{2}^{*}\left(q_{1}, \theta\right)}{\partial q_{1}}
\end{gathered}
$$

where $\frac{\partial q_{2}^{*}\left(q_{1}, \theta\right)}{\partial q_{1}}$ is well-defined from the fact that $T_{2}(\cdot)$ is twice differentiable. Inserting into (35) yields for a symmetric equilibrium such that $q_{1}(\theta)=q_{2}(\theta)=q(\theta)$ :

$$
\begin{aligned}
& q(\theta) P^{\prime}(2 q(\theta))+P(2 q(\theta))=C^{\prime}(2 q(\theta))\left(\theta+\frac{F(\theta)}{f(\theta)}\right) \\
& +\frac{\theta C^{\prime \prime}(2 q(\theta))}{T^{\prime \prime}(q(\theta))-\theta C^{\prime \prime}(2 q(\theta))}\left(\frac{F(\theta)}{f(\theta)}-q(\theta) P^{\prime}(2 q(\theta))\right) .
\end{aligned}
$$

However, in a symmetric equilibrium, $T^{\prime}(q(\theta))=\theta C^{\prime}(2 q(\theta))$ for all $\theta$. Differentiating w.r.t. $\theta$ yields:

$$
\left(T^{\prime \prime}(q(\theta))-\theta C^{\prime \prime}(2 q(\theta))\right) \dot{q}(\theta)=\theta C^{\prime \prime}(2 q(\theta)) \dot{q}(\theta)+C^{\prime}(2 q(\theta)) .
$$

Inserting into (36) yields (11).

Once we have determined $q(\theta)$, the determination of $\theta_{0}$ is found by solving the program

$$
\max _{\theta_{0}} \int_{\underline{\theta}}^{\theta_{0}} V(q(\theta), \theta) f(\theta) d \theta .
$$


Differentiating under the integral gives us the condition that $\theta_{0}$ solves $V\left(q\left(\theta_{0}\right), \theta_{0}\right)=0$ is a solution exists in $\Theta$ and $\theta=\bar{\theta}$ otherwise.

- We now establish the bounds on $q(\theta)$. We prove that $q(\theta) \in\left[\tilde{q}^{c}(\theta), q^{b}(\theta)\right]$ for all $\theta$. First we show that if $q(\underline{\theta}) \in\left[q^{c}(\underline{\theta}), q^{b}(\underline{\theta})\right]$, then the solution to (11) starting from this point remains in the set $\left[\tilde{q}^{c}(\theta), q^{b}(\theta)\right]$ for all $\theta$.

Suppose that there exists $\hat{\theta}$, the first value of $\theta$ greater than $\underline{\theta}$, such that $q(\hat{\theta})=q^{b}(\hat{\theta})$. We have then:

$$
\dot{q}(\hat{\theta})=-\frac{C^{\prime}}{2 \theta C^{\prime \prime}}<\dot{q}^{b}(\hat{\theta})=-\frac{C^{\prime}}{2 \theta C^{\prime \prime}-2 P^{\prime}}<0 .
$$

Hence, $q(\theta)>q^{b}(\theta)$ for $\theta \in(\hat{\theta}-\epsilon, \hat{\theta})$ where $\epsilon$ is small enough. A contradiction.

Suppose that there exists $\hat{\theta}$, the first value of $\theta$ greater than $\underline{\theta}$, such that $q(\hat{\theta})=\tilde{q}^{c}(\hat{\theta})$. We have then:

$$
\dot{q}(\hat{\theta})=0>\dot{q}^{c}(\hat{\theta})
$$

when $\frac{d}{d \theta}\left(\frac{F(\theta)}{f(\theta)}\right)>0$, and $C(\cdot)$ convex. Hence, $q(\theta)<\tilde{q}^{c}(\theta)$ for $\theta \in(\hat{\theta}-\epsilon, \hat{\theta})$ where $\epsilon$ is small enough. A contradiction.

Second, we prove that no solution exists with $q(\underline{\theta}) \notin\left[q^{c}(\underline{\theta}), q^{b}(\underline{\theta})\right]$. Consider (36) evaluated at $\underline{\theta}$. The following two relationships emerge (where the inequalities follow from applying the agent's local second-order condition):

$$
\begin{gathered}
q(\underline{\theta}) P^{\prime}(2 q(\underline{\theta}))+P(2 q(\underline{\theta}))-\underline{\theta} C^{\prime}(2 q(\underline{\theta}))=-q(\underline{\theta}) P^{\prime}(2 q(\underline{\theta}))\left(\frac{\underline{\theta} C^{\prime \prime}(2 q(\underline{\theta}))}{T^{\prime \prime}(q(\underline{\theta}))-\underline{\theta} C^{\prime \prime}(2 q(\underline{\theta}))}\right)<0, \\
P(2 q(\underline{\theta}))-\underline{\theta} C^{\prime}(2 q(\underline{\theta}))=q(\underline{\theta}) P^{\prime}(2 q(\underline{\theta}))\left(\frac{\underline{\theta} C^{\prime \prime}(2 q(\underline{\theta}))}{T^{\prime \prime}(q(\underline{\theta}))-\underline{\theta} C^{\prime \prime}(2 q(\underline{\theta}))}+1\right)>0 .
\end{gathered}
$$

Hence, $q^{c}(\underline{\theta}) \leq q(\underline{\theta}) \leq q^{b}(\underline{\theta})$.

- We now turn to the global concavity of the agent's problem: Using (11), we have:

$$
\begin{aligned}
& T^{\prime \prime}(q(\theta))-\theta C^{\prime \prime}(2 q(\theta))=\frac{\theta C^{\prime \prime}(2 q(\theta))}{q(\theta) P^{\prime}\left(2 q(\theta)+P(2 q(\theta))-C^{\prime}(2 q(\theta))\left(\theta+\frac{F(\theta)}{f(\theta)}\right)\right.} \\
& \times\left(-q(\theta) P^{\prime}(2 q(\theta))+\frac{F(\theta)}{f(\theta)} C^{\prime}(2 q(\theta))\right) .
\end{aligned}
$$

Since $q(\theta)>\tilde{q}^{c}(\theta)$, the R.H.S. above is negative. Moreover, $\left|T^{\prime \prime}(q(\theta))-\theta C^{\prime \prime}(2 q(\theta))\right| \geq \theta C^{\prime \prime}(2 q(\theta))$ since $P(2 q(\theta)) \geq \theta C^{\prime}(2 q(\theta))$ when $q(\theta) \leq q^{b}(\theta)$. 
The facts that $T^{\prime \prime}(q(\theta))-\theta C^{\prime \prime}(2 q(\theta)) \leq 0$ and that $\left|T^{\prime \prime}(q(\theta))-\theta C^{\prime \prime}(2 q(\theta))\right| \geq \theta C^{\prime \prime}(2 q(\theta))$ prove that the Hessian of the type $\theta$-agent's problem is negative semi-definite at $(q(\theta), q(\theta))$ for any $\theta$ and any equilibrium schedule $q(\theta)$. Hence, the agent's objective function is locally concave at this point. To have global concavity of the agent's problem, we need more. This Hessian must be negative semi-definite at all pairs $\left(q_{1}, q_{2}\right)$. To prove this, let us write the equilibrium nonlinear price as $T(q)=\int_{q\left(\theta_{0}\right)}^{q} \theta(z) C^{\prime}(2 z) d z+\theta_{0} C\left(2 q\left(\theta_{0}\right)\right) / 2$ where $\theta(q)$ is the inverse function of an equilibrium schedule $q(\theta)$ which is well defined since $q(\cdot)$ is decreasing. An agent with type $\theta$ wants to maximize $J\left(q_{1}, q_{2}\right)=T\left(q_{1}\right)+T\left(q_{2}\right)-\theta C\left(q_{1}+q_{2}\right)$. The corresponding first-order conditions are:

$$
\begin{aligned}
& J_{1}\left(q_{1}, q_{2}\right)=\theta\left(q_{1}\right) C^{\prime}\left(2 q_{1}\right)-\theta C^{\prime}\left(q_{1}+q_{2}\right)=0 \\
& J_{2}\left(q_{1}, q_{2}\right)=\theta\left(q_{2}\right) C^{\prime}\left(2 q_{2}\right)-\theta C^{\prime}\left(q_{1}+q_{2}\right)=0 .
\end{aligned}
$$

Clearly, those first-order conditions hold as equalities when $q_{1}=q_{2}=q(\theta)$. Moreover, we have:

$$
J_{11}\left(q_{1}, q_{2}\right)=2 \theta\left(q_{1}\right) C^{\prime \prime}\left(2 q_{1}\right)+\dot{\theta}\left(q_{1}\right) C^{\prime}\left(2 q_{1}\right)-\theta C^{\prime \prime}\left(q_{1}+q_{2}\right)
$$

and

$$
J_{12}\left(q_{1}, q_{2}\right)=-\theta C^{\prime \prime}\left(q_{1}+q_{2}\right) .
$$

We observe that $\alpha\left(q_{1}\right)=2 \theta\left(q_{1}\right) C^{\prime \prime}\left(2 q_{1}\right)+\dot{\theta}\left(q_{1}\right) C^{\prime}\left(2 q_{1}\right)$ has the opposite sign as $2 \frac{\theta C^{\prime \prime}(2 q(\theta))}{C^{\prime}(2 q(\theta))} \dot{q}(\theta)+1$ for all $q_{1}$ in the range of $q(\cdot)$. This last term is equal to

$$
\frac{-P(2 q(\theta))+\theta C^{\prime}(2 q(\theta))}{P(2 q(\theta))+2 q(\theta) P^{\prime}(2 q(\theta))-\left(\theta+2 \frac{F(\theta)}{f(\theta)}\right) C^{\prime}(2 q(\theta))}
$$

which is positive since $\tilde{q}^{c}(\theta) \leq q(\theta) \leq q^{b}(\theta)$. Hence $J_{11}\left(q_{1}, q_{2}\right)<0$ for all $\left(q_{1}, q_{2}\right)$. Similarly, $J_{22}\left(q_{1}, q_{2}\right)<0$ for all $\left(q_{1}, q_{2}\right)$. Moreover, we have

$$
J_{11}\left(q_{1}, q_{2}\right) J_{22}\left(q_{1}, q_{2}\right)-\left(J_{12}\left(q_{1}, q_{2}\right)\right)^{2}=\alpha\left(q_{1}\right) \alpha\left(q_{2}\right)-\theta C^{\prime \prime}\left(q_{1}+q_{2}\right)\left(\alpha\left(q_{1}\right)+\alpha\left(q_{2}\right)\right)>0
$$

since $\alpha\left(q_{i}\right)<0$ for $i=1,2$.

For outputs which lie outside the range of the equilibrium schedule $q(\theta)$, i.e., which are greater than $q(\underline{\theta})$ the equilibrium tariff is extended in a quadratic (and continuously differentiable) way so that these conditions also hold. 
- We now check that the indirect utility function vis-à-vis either principal satisfies the SpenceMirrlees single-crossing property, $u_{i \theta}^{i}(q, \theta) \leq 0$ for all $q$ and $i=1,2$. In fact, we have, for any equilibrium point $q\left(\theta^{\prime}\right)$ :

$$
u_{i \theta}^{i}\left(q\left(\theta^{\prime}\right), \theta\right)=-C^{\prime}\left(q\left(\theta^{\prime}\right)+q_{-i}^{*}\left(q\left(\theta^{\prime}\right), \theta\right)\right)\left(1+\frac{\partial q_{-i}^{*}\left(q\left(\theta^{\prime}\right), \theta\right)}{\partial q}\right) .
$$

Omitting arguments, and using symmetry, the bracketed term in the RHS above is

$$
-\frac{C^{\prime}\left(2 q\left(\theta^{\prime}\right)+2 \theta^{\prime} C^{\prime \prime}\left(2 q\left(\theta^{\prime}\right)\right) \dot{q}\left(\theta^{\prime}\right)\right.}{C^{\prime}\left(2 q\left(\theta^{\prime}\right)+\theta^{\prime} C^{\prime \prime}\left(2 q\left(\theta^{\prime}\right)\right) \dot{q}\left(\theta^{\prime}\right)\right.} .
$$

Using (11), this RHS becomes:

$$
\left(\frac{P\left(2 q\left(\theta^{\prime}\right)\right)-\theta^{\prime} C^{\prime}\left(2 q\left(\theta^{\prime}\right)\right)}{q\left(\theta^{\prime}\right) P^{\prime}\left(2 q\left(\theta^{\prime}\right)\right)-\frac{F\left(\theta^{\prime}\right)}{f\left(\theta^{\prime}\right)} C^{\prime}\left(2 q\left(\theta^{\prime}\right)\right)}\right)
$$

which is negative since $q\left(\theta^{\prime}\right) \leq q^{b}\left(\theta^{\prime}\right)$ for all $\theta^{\prime}$. Because $T(\cdot)$ is extended over the whole real line in a quadratic way, the latter inequality holds for all $q$ even those for which there does not exist $\theta^{\prime}$ such that $q=q\left(\theta^{\prime}\right)$.

- Let us provide conditions ensuring the concavity of $V\left(q_{1}, \theta\right)$ with respect to $q_{1}$. We can rewrite:

$$
V\left(q_{1}, \theta\right)=q_{1} P\left(q_{1}+q_{2}^{*}\left(q_{1}, \theta\right)\right)+T_{2}\left(q_{2}^{*}\left(q_{1}, \theta\right)\right)-C\left(q_{1}+q_{2}^{*}\left(q_{1}, \theta\right)\right)\left(\theta+\frac{F(\theta)}{f(\theta)}\right) .
$$

Differentiating twice and taking into account (1) yields:

$$
\begin{gathered}
\frac{\partial^{2} V}{\partial q_{1}^{2}}\left(q_{1}, \theta\right)=\left(1+\frac{\partial q_{2}^{*}\left(q_{1}, \theta\right)}{\partial q_{1}}\right) \times \\
\left(2 P^{\prime}\left(q_{1}+q_{2}^{*}\left(q_{1}, \theta\right)\right)+q_{1} P^{\prime \prime}\left(q_{1}+q_{2}^{*}\left(q_{1}, \theta\right)\right)\left(1+\frac{\partial q_{2}^{*}\left(q_{1}, \theta\right)}{\partial q_{1}}\right)^{2}-\frac{F(\theta)}{f(\theta)}\left(1+\frac{\partial q_{2}^{*}\left(q_{1}, \theta\right)}{\partial q_{1}}\right) C^{\prime \prime}\left(q_{1}+q_{2}^{*}\left(q_{1}, \theta\right)\right)\right) \\
-\theta\left(1+\frac{\partial q_{2}^{*}\left(q_{1}, \theta\right)}{\partial q_{1}}\right) C^{\prime \prime}\left(q_{1}+q_{2}^{*}\left(q_{1}, \theta\right)\right) \\
+\frac{\partial^{2} q_{2}^{*}\left(q_{1}, \theta\right)}{\partial q_{1}^{2}}\left(q_{1} P^{\prime}\left(q_{1}+q_{2}^{*}\left(q_{1}, \theta\right)\right)-\frac{F(\theta)}{f(\theta)} C^{\prime}\left(q_{1}+q_{2}^{*}\left(q_{1}, \theta\right)\right)\right) .
\end{gathered}
$$

But $\frac{\partial q_{2}^{*}\left(q_{1}, \theta\right)}{\partial q_{1}}=\frac{\theta C^{\prime \prime}\left(q_{1}+q_{2}^{*}\left(q_{1}, \theta\right)\right)}{T^{\prime \prime}\left(q_{2}^{*}\left(q_{1}, \theta\right)\right)-\theta C^{\prime \prime}\left(q_{1}+q_{2}^{*}\left(q_{1}, \theta\right)\right)}$ where $T(\cdot)$ is a symmetric equilibrium nonlinear price which satisfies the conditions (22) and (23) and thus $\left(1+\frac{\partial q_{2}^{*}\left(q_{1}, \theta\right)}{\partial q_{1}}\right)>0$ (obtained from (22)). The first term on the RHS above is negative since $\left(1+\frac{\partial q_{2}^{*}\left(q_{1}, \theta\right)}{\partial q_{1}}\right)>0, P^{\prime}<0$ and $P^{\prime \prime} \leq 0$. The second term is negative if $\frac{\partial^{2} q_{2}^{*}\left(q_{1}, \theta\right)}{\partial q_{1}^{2}} \geq 0$. 
- Finally, principal 1 wants to deal with type $\theta$ as long as the the virtual surplus he draws from that is positive. This means that $\theta^{*}$, the upper bound of that set is at the corner $\bar{\theta}$ when:

$$
V(q(\bar{\theta}), \bar{\theta})>0
$$

Using that, in a symmetric equilibrium $2 T\left(q(\bar{\theta})=\theta^{*} C(2 q(\bar{\theta}))\right.$, we must have:

$$
q(\bar{\theta}) P(2 q(\bar{\theta}))-\left(\frac{\bar{\theta}}{2}+\frac{F(\bar{\theta})}{f(\bar{\theta})}\right) C(2 q(\bar{\theta}))>0 .
$$

